\title{
Electrically active magnetic excitations in antiferromagnets
}

\author{
(Review Article)
}

\author{
V.N. Krivoruchko \\ O.O. Galkin Donetsk Institute for Physics and Technology, National Academy of Science of Ukraine \\ 72 R. Luxemburg Str., Donetsk 83114, Ukraine \\ E-mail: krivoruc@krivoruc.fti.ac.donetsk.ua; krivoruc@gmail.com
}

Received March 20, 2012

\begin{abstract}
The magnetic resonance operation by electric field is highly nontrivial but the most demanding function in the future spin-electronics. Recently observed in a variety of multiferroics materials named the collective electrically active magnetic excitations, frequently referred to as "electromagnons", reveal a possible way to implement such a function. Experimental advances in terahertz spectroscopy of electromagnons in multiferroics as well as related theoretical models are reviewed. The earlier theoretical works, where the existence of electric-dipole active magnetic excitations in antiferro- and ferrimagnets with collinear spin structure has been predicted, are also discussed. Multi-sublattice magnets with electrically active magnetic excitations at room temperature give a direct possibility to transform one type of excitation into another in a terahertz time-domain. This is of crucial importance for the magnon-based spintronics as only the short-wavelength exchange magnons allow the signal processing on the nanoscale distance.
\end{abstract}

PACS: 75.80.+q Magnetomechanical effects, magnetostriction;

75.40.Gb Dynamic properties (dynamic susceptibility, spin waves, spin diffusion, dynamic scaling, etc.);

76.50.+g Ferromagnetic, antiferromagnetic, and ferrimagnetic resonances; spin-wave resonance.

Keywords: ferrimagnet, electromagnons, spintronics, electron paramagnetic resonance.

\section{Contents}

1. Introduction. 1018

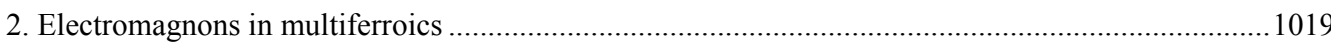

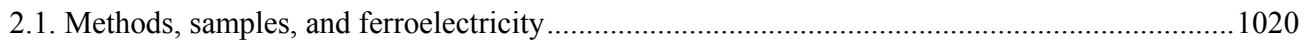

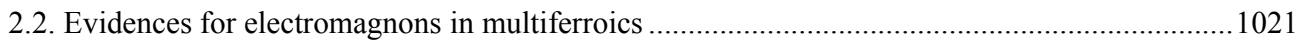

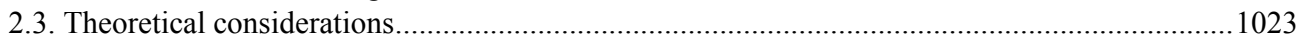

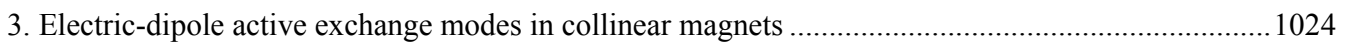

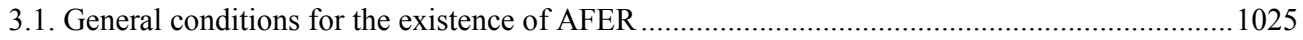

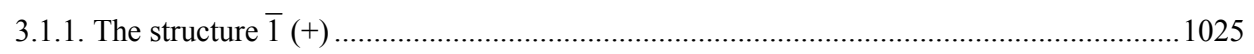

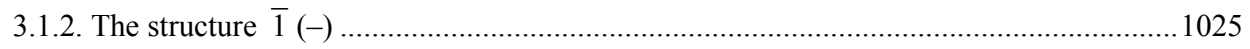

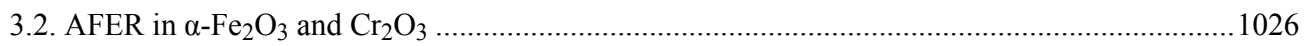

4. Electric-dipole active magnetic resonances in ferrimagnets......................................................... 1028

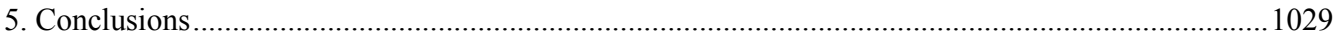

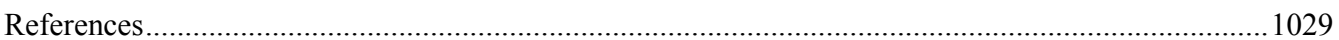

\section{Introduction}

In 1961 a new effect has been discovered in spectroscopy of solid state, namely, the applied electric field effect on the electron spin resonance [1]. This effect is manifested by a linear on electric field $\mathbf{E}$ splitting of absorption lines in the electron paramagnetic resonance spectrum and evidences the external electric field effect on the energy levels of magnetic ion. Another manifestation of the electric effect, discovered in 1962 [2], was the so-called paraelectric resonance, i.e., an induction of transitions between magnetic levels of paramagnetic ions by a high-frequency electric field. Differently speaking, the magnetic resonance could be observed by placing the sample into the ac electric field antinodes instead of ac magnetic field ones. In this way a possibility to excite transitions forbidden for a conventional magnetic resonance was demonstrated. In further investigations, electrically induced transitions between spin levels were detected for different magnetic ions and explored in a number of reports (see review [3] and textbooks [4,5]). 
It should be noted that the possibility to induce the magnetic resonance transitions by an alternating electric field follows already from the spin Hamiltonian of the form

$$
\mathcal{H}=E_{i}\left(R_{i j k} S_{j} S_{k}+T_{i j k} H_{j} S_{k}\right) ; \quad i, j, k=x, y, z,
$$

which is used for description of linear electric field effects (here $\mathbf{S}$ is the spin operator, $\mathbf{H}$ stands for external magnetic field) [3-5]. From viewpoint of theory, there are no principal differences between the static and alternating field; it is only important that related magnetoelectric constants, $R_{i j k}$ and $T_{i j k}$, must be nonzero as well as the corresponding matrix elements. Let us remind that linear electric effects are feasible (the magnetoelectric constants are nonzero) for paramagnetic ions in the non-center-symmetric positions [3-5]. Obviously, in concentrated magnetic compounds the existence of ions with electric-dipole active magnetic transitions yields collective magnetic excitations. But the limited experimental possibilities, such as a hard frequency range - terahertz and even optic frequency range - impede progress in the field.

Terahertz $(\mathrm{THz})$ frequency range (region of frequency from $0.1 \mathrm{THz}$ up to $10 \mathrm{THz}$, energy scale $1 \mathrm{THz} \approx 4 \mathrm{meV}$ ) involves the short-wave part of millimeter range, all submillimeter region and a part of far infrared range (wave length from $3 \mathrm{~mm}$ up to $0.03 \mathrm{~mm}$ ). This region of electromagnetic radiation remains unexplored since long ago. At the same time, the radiation in this frequency range possesses a set of specific characteristics, which makes it rather promising for applications in fundamental and applied physics, chemistry, biology, medicine and so on (for more details see recent reviews [6,7] and references therein). At present, a so-called "THz-gap" is actively mastered by traditional vacuum electronics as well as by optoelectronics.

During the last years, the $\mathrm{THz}$ radiation was found to be very sensitive and informative tool for investigation quasiparticle excitations in solid state in this frequency/energy range - polaritons, excitons, phonons and others. In fact, nowadays the $\mathrm{THz}$ time-domain spectroscopy enters into toolset of many physical laboratories [6,7]. A class of the system where $\mathrm{THz}$ spectroscopy has already displayed its unique performances is the so-called multiferroics - materials, where the magnetic order and ferroelectric polarization coexist. Exactly in multiferroics it was succeeded to detect by the $\mathrm{THz}$ spectroscopy technique and identify a new type of collective electrically active magnetic excitation frequently termed as "electromagnons".

The first part of this paper (Sec. 2) presents an overview of recent experimental advances in $\mathrm{THz}$ spectroscopy of electromagnons in multiferroics. The proposed theoretical models of electromagnons origin in multiferroics are also discussed. Next part (Sec. 3) summarizes the earlier theoretical works where existence of electric-dipole active magnetic excitations in antiferromagnets has been predicted. The idea was put forward for the first time by Ukrainian physicists in 1988 in series of publications, in particular, with Victor Eremenko participation. Here, the general conditions for appearance of electrically active magnetic oscillations in magnetic structures which ground state is collinear in the exchange approximation are formulated. As examples, the four-sublattices hematite $\left(\alpha-\mathrm{Fe}_{2} \mathrm{O}_{3}\right)$ and chromium (III) oxide $\left(\mathrm{Cr}_{2} \mathrm{O}_{3}\right)$, are considered. It is shown that $\mathrm{THz}$ spectroscopy could be a fruitful, and sometimes even sole, experimental tool for investigation magnetic excitations in these antiferromagnets. In Sec. 4, the electric-dipole active magnetic resonances in compounds possessing both spontaneous magnetization and electric polarization are briefly discussed.

Possibility to transform magnetic excitation into electric signal and vice-versa is highly nontrivial but the most demanding function in the future spin-electronics. Due to magnetic ordering well above room temperature, the multisublattice magnets with electrically active magnetic excitations give an opportunity for implementation of such function and thus are promising candidates for future applications in spin based electronics.

\section{Electromagnons in multiferroics}

Materials with the simultaneous occurrence of magnetism and ferroelectricity, which are frequently referred to as multiferroics [8] (in earlier Russian literature often named as seignetto-magnets), are characterized by a strong coupling between magnetic order and electric polarization. Such perovskite manganites as $\mathrm{TbMnO}_{3}$ and $\mathrm{GdMnO}_{3}$ are examles of typical family members of multiferroics with a ferroelectricity induced by magnetic order. Exactly for these compounds, apparently for the first time, the strong experimental evidence was obtained of new type of magnetic excitations, namely the electric-dipole active magnons or electromagnons $[9,10]$. These new elementary excitations were manifested in the noncommensurate spin ordered phase by additional absorption peaks in the dielectric constant spectrum $\varepsilon(\omega)$ within the THz frequency region. These peaks vanished in applied magnetic field, which produced a commensurate spin order. Such a behavior points to the magnetic origin of excitations.

It should be noted here that the existence of electricdipole active magnons had been theoretically predicted much earlier than the electromagnons were first observed. In the end of 60th - beginning the 70th years, the theoreticians V.G. Bar'yakhtar and I.E. Chupis have demonstrated in a number of works $[11,12]$ that in seignetto-magnets, the liner oscillations of electric and magnetic dipoles are coupled. Eventually, oscillations of magnetic moments could be caused by electric field and, vice-versa, the electric-dipole excitations by magnetic field. This new elementary excitations, called by the authors the seignette-magnons, appear as magnetic resonances in the dielectric constant spectrum $\varepsilon(\omega)$ in a response to the electric field component of light $E_{\omega}$. This is in contrast to the case of the 
magnetic resonances appearing in the magnetic permeability spectrum $\mu(\omega)$ as a result of the spin excitations driven by the magnetic component of light $H_{\omega}$. The concept proposed in Refs. 11, 12 was developed in following publications, the review of which is given in Ref. 13. Unfortunately, as already was mentioned, the limited experimental facilities postponed the full-scale investigations in the field and a discovery of seignette-magnons.

Renewed attention to multiferroics has been inspired by discovery of the electric polarization flop by applying magnetic field in a perovskite manganite [14]. Obviously, the dynamical interplay between magnetic and electric excitations has been reasonably expected for these materials, as well. Indeed, soon after the work [14], the observation of electric-dipole active magnetic excitations were reported for $\mathrm{TbMnO}_{3}$ and $\mathrm{GdMnO}_{3}$ as far-infrared absorption near $2.5 \mathrm{meV}[9,10]$.

\subsection{Methods, samples and ferroelectricity}

Typically, in experiments with $\mathrm{THz}$ radiation, the value of the complex optical constant $n(\omega)=\sqrt{\varepsilon(\omega) \mu(\omega)}$ is determined from the measured transmittance spectrum. In this frequency region the contribution of magnetic permeability $\mu(\omega)$ to $n(\omega)$ in most cases is negligible and frequently $\mu(\omega) \approx 1$ is assumed. The transmittance experiments at $\mathrm{THz}$ frequencies can be carried out in a MachZehnder interferometer arrangement [15] which allows measurements of amplitude and phase shift in a geometry with controlled polarization of the radiation. The absolute values of the complex dielectric permittivity $\varepsilon=\varepsilon_{1}+i \varepsilon_{2}$ are determined directly from the measured spectra using the Fresnel optical formulas for transmittance $T=|t|^{2}$ of a plane-parallel sample:

$$
t=\sqrt{T} \exp \left(i \varphi_{T}\right)=\frac{\left(1-r^{2}\right) t_{1}}{1-r^{2} t_{1}^{2}},
$$

where $r=(\sqrt{\varepsilon}-1) /(\sqrt{\varepsilon}+1)$ and $t_{1}=\exp (-2 \pi i \sqrt{\varepsilon} d / \lambda)$. Here $r$ is the reflection amplitude of a thick sample, $t_{l}$ is the "pure" transmission amplitude, $d$ is the sample thickness, $\lambda$ is the radiation wavelength, and $\varphi_{T}$ is the phase shift in the transmittance.

The transmittance experiment with different experimental geometries and varied polarization of the incident radiation usually allows to separate unambiguously the magnetic and dielectric contributions to the measured spectra. However, in some cases both contributions are equally strong and four independent experiments are necessary to extract both, the dielectric permittivity $\varepsilon(\omega)$ and the magnetic permeability $\mu(\omega)$.

A majority of existing experimental results are obtained on single (or poly-) crystalline samples of $\mathrm{RMnO}_{3}$, and a brief discussion of the magnetic phase diagram of these compounds is not out of place.
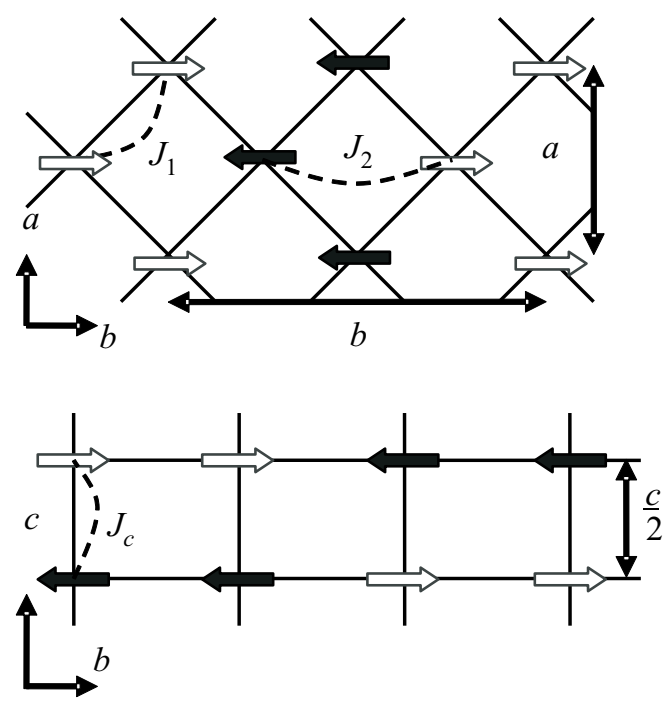

Fig. 1. The schematic spin configuration of the $E$-type state in $a b$ and $b c$ planes.

In many of the manganite materials with perovskite structure, $\mathrm{RMnO}_{3}$, the nearest-neighbor ferromagnetic exchange, $J_{1}$, is weak relative to the next-neighbor antiferromagnetic interaction $J_{2}$ (see Fig. 1). As the result, the magnetic order is sensitive to the $\mathrm{Mn}-\mathrm{O}-\mathrm{Mn}$ bond angle or, equivalently, to the ionic radius of $\mathrm{R}$. The phase diagram of $\mathrm{RMnO}_{3}$ consists of the $A$-type (ferromagnetic $a b$-plane antiferromagnetically ordered), $a b$-cycloidal (Fig. 2) and $E$-type $(\uparrow \uparrow \downarrow \downarrow)$ (Fig. 1) states. When $J_{2} /\left|J_{1}\right|>0.5$, minimum magnetic energy achieves for cycloidal structure with noncommensurate period. In $\mathrm{RMnO}_{3}$ crystals with $\mathrm{R}=\mathrm{Tb}, \mathrm{Dy}$, $\mathrm{Eu}_{1-x} \mathrm{Y}_{x}$ a cycloidal structure is realized within $a b$ plane. Such magnetic order induces a nonzero static electric polarization $\mathbf{P} \| a b$ plane. The magnetically induced ferroelectricity has been explained by the inverse DzyaloshinskiiMoriya (DM) interaction [16-18], in which the microscopic polarization is expressed as $\mathbf{P}_{A} \sim \mathbf{e}_{n m} \times\left(\mathbf{S}_{n} \times \mathbf{S}_{m}\right)$ for a pair of neighboring spins, $\mathbf{S}_{n}$ and $\mathbf{S}_{m}$, with unit vector $\mathbf{e}_{n m}$ connecting them.

In perovskites with rare earth ions of smaller radius ( $\mathrm{R}=\mathrm{Ho}, \mathrm{Tm}, \mathrm{Yb}, \mathrm{Lu}, \mathrm{Y}_{1-x} \mathrm{Lu}_{x}$ ) minimum magnetic energy achieves for $E$-type magnetic order in $a b$ plane, hosting also the ferroelectricity. It was shown that the ferroelectricity in the $E$-type phase originates from the symmetric exchange term expressed as $\mathbf{P}_{S} \sim \boldsymbol{\pi}_{n m}\left(\mathbf{S}_{n} \cdot \mathbf{S}_{m}\right)$ which poten-

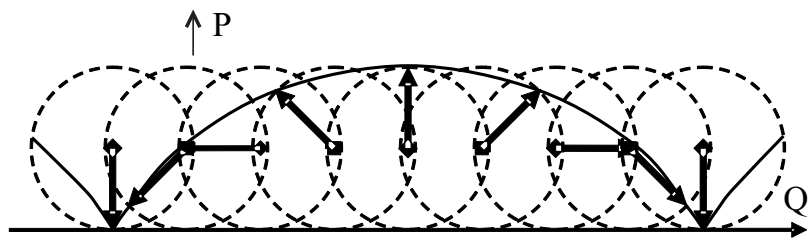

Fig. 2. Cycloidal spin spiral structure; the spins rotate around an axis normal to the spiral wave vector $\mathbf{Q}$. The induced electric polarization $\mathbf{P}$ is normal to both the wave vector and the spin rotation axis: $\mathbf{P} \perp \mathbf{Q}$. 
tially produces larger spontaneous polarization than antisymmetric DM mechanism [19-21]. Indeed, it was found $[20,21]$ that the electric polarization of the $E$-type phase is dominated by the symmetric spin exchange $\left(\mathbf{S}_{n} \cdot \mathbf{S}_{m}\right)$ and is more than ten times larger that of the $b c$-cycloidal phase dominated by the antisymmetric one $\sim\left(\mathbf{S}_{n} \times \mathbf{S}_{m}\right)$. The magnetoelectric response is also enhanced near the bicritical phase boundary between these multiferroic phases of different origins. (Note for reference, the largest magnetically induced electrical polarization was documented recently in $\mathrm{CaMn}_{7} \mathrm{O}_{12}$ [22]. The value of $P_{S}=2870 \mu \mathrm{C} / \mathrm{m}^{2}$ for $\mathrm{CaMn}_{7} \mathrm{O}_{12}$ is four time large than a spontaneous polarization in $\mathrm{TbMnO}_{3}$.)

\subsection{Evidences for electromagnons in multiferroics}

Pimenov et al. have measured the optical spectra of $\mathrm{TbMnO}_{3}$ and $\mathrm{GdMnO}_{3}$ in the energy range $0.4-4.8 \mathrm{meV}$ $[9,10]$. At low temperature in zero magnetic field, they found a single peak-structure in the imaginary part of $\varepsilon(\omega)$ at $2.9 \mathrm{meV}$ for $\mathrm{TbMnO}_{3}$ and at $2.5 \mathrm{meV}$ for $\mathrm{GdMnO}_{3}$ (see Fig. 3). These absorptions were shown to be permitted if $\mathbf{E}_{\omega} \| a$ axis and disappeared when the $b c$-spiral order is transformed to the $A$-type antiferromagnetic order by applying the external magnetic field along $c$ axis.

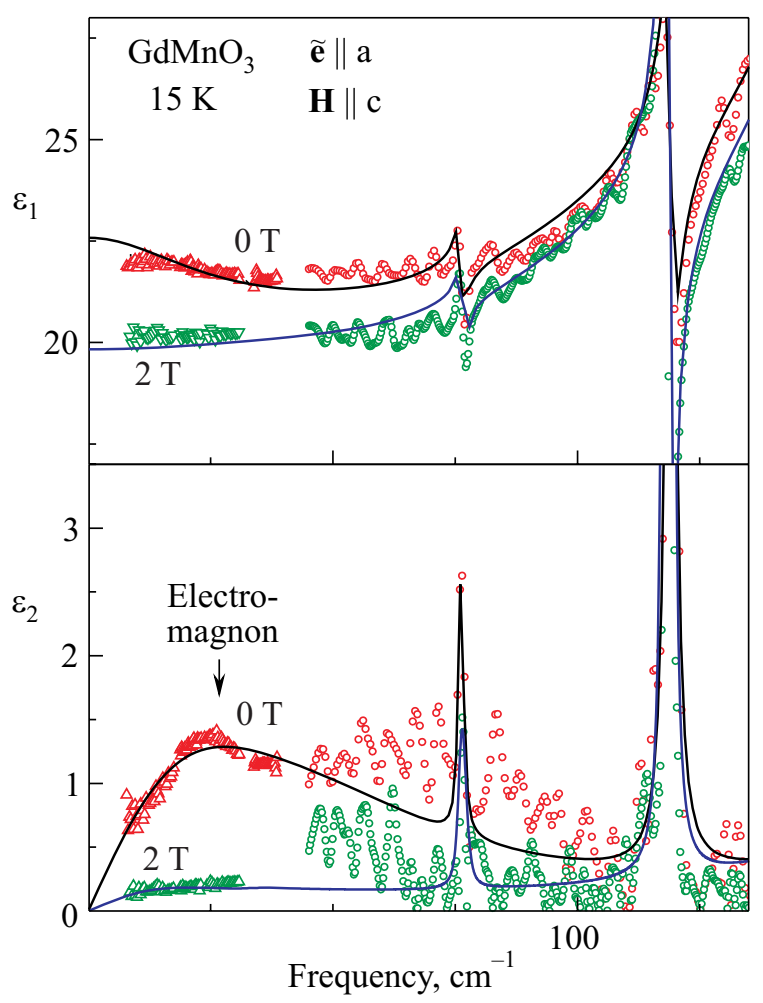

Fig. 3. Spectra of the $a$-axis dielectric permittivity of $\mathrm{GdMnO}_{3}$ in the $\mathrm{THz}$ frequency range at 0 and $2 \mathrm{~T}$. Upper panel: real part of the dielectric permittivity; lower panel: imaginary part. Triangles represent the results from the $\mathrm{THz}$ transmittance. Circles - spectra obtained via Kramers-Kronig analysis of the reflectance. Electromagnon is detected as a broad peak at $25 \mathrm{~cm}^{-1}$. (Reproduced from Ref. 10.)
Pioneering works $[9,10]$ stimulated a series of publications concerning the electric-dipole active spin dynamics in crystals of different symmetry with noncommensurate magnetic structure and multiferroic properties: $\mathrm{RMnO}_{3}$ [23-44], $\mathrm{BiFeO}_{3}$ [45,46], $\mathrm{RMn}_{2} \mathrm{O}_{5}$ [47-49], $\mathrm{Cu}(\mathrm{Fe}, \mathrm{Ca}) \mathrm{O}_{2}$ [50], $\mathrm{CuFe}_{1-x} \mathrm{Ga}_{x} \mathrm{O}_{2}$ [51,52], and $\mathrm{Ba}_{2} \mathrm{CoGeO}_{7}$ [53]. These investigations revealed new excitations in $\mathrm{THz}$ time domain region and posed a set of questions.

Note here that different crystal imperfections (polycrystalline structure of a sample, ferroelectric domain walls, etc.) and (especially) impurity of rare-earth ions also interact with the electric component of light and give contributions to the $\varepsilon(\omega)$ spectrum $[54,55]$. To avoid these problems, at least partly, the systems $\mathrm{RMnO}_{3}$ where $\mathrm{Mn}^{3+}$ ion is the only magnetic ion are found to be the most relevant for revealing the physics of electromagnons in multiferroics.

Leaving behind the historical sequence of experimental data obtaining, let us concentrate on the settled ones.

Most comprehensive data are obtained on the compounds with $\mathrm{R}=\mathrm{Y}, \mathrm{Lu}, \mathrm{Y}_{1-x} \mathrm{Lu}_{x}, \mathrm{Eu}, \mathrm{Eu}_{1-x} \mathrm{Y}_{x}$. Figures 4 and 5 illustrate the results on $\mathrm{THz}$ spectroscopy of electromagnons in perovskites manganites $\mathrm{RMnO}_{3}$ with $\mathrm{R}=$ $=\mathrm{Y}_{1-x} \mathrm{Lu}_{x}, \mathrm{Eu}_{1-x} \mathrm{Y}_{x}$ [44]. In Fig. 4, the representative spectra are displayed of the $A$-type, $a b$-cycloidal and $E$-type

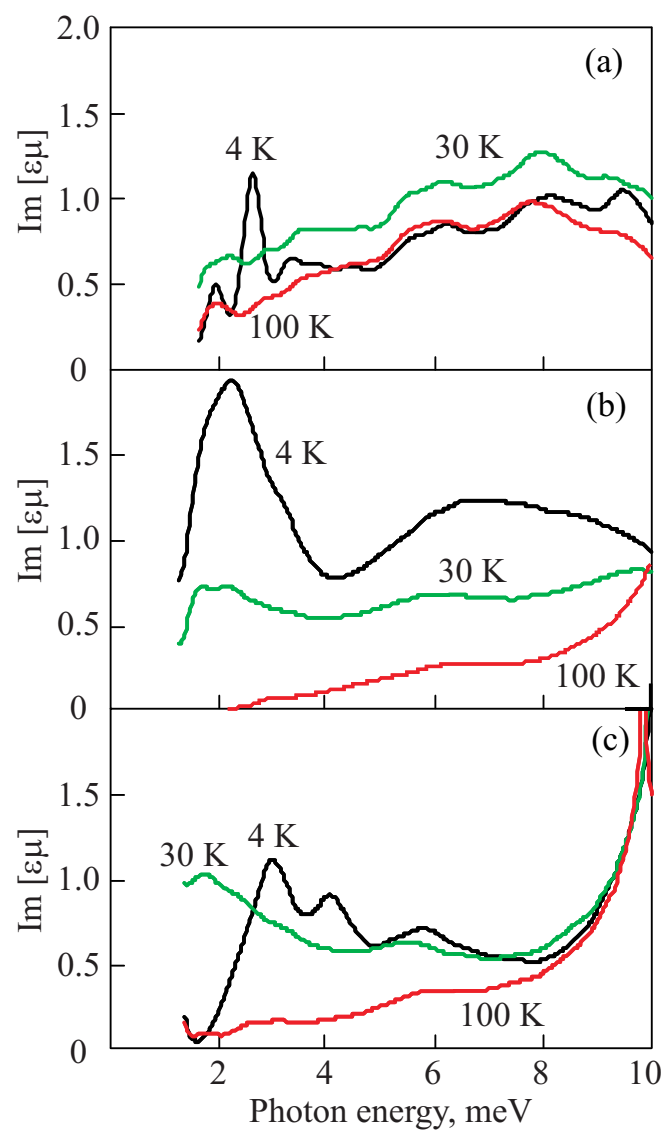

Fig. 4. The Im $[\varepsilon \mu]$ spectra for: (a) $A$-type (collinear in $a b$ plane), (b) $a b$-cycloidal, and (c) $E$-type ( $\uparrow \downarrow \downarrow$ in $a b$ plane) spin phases of $\mathrm{Eu}_{0.8} \mathrm{Y}_{0.2} \mathrm{MnO}_{3}, \mathrm{Eu}_{0.4} \mathrm{Y}_{0.6} \mathrm{MnO}_{3}$, and $\mathrm{Y}_{0.5} \mathrm{Lu}_{0.5} \mathrm{MnO}_{3}$, respectively. (Reproduced from Ref. 44.) 
states. The plateau-like absorption bands are observed in the higher- $T$ collinear sinusoidal phase for all the compounds in addition to the tail of the higher-lying phonon modes; this broadened band has been assigned to the precursory electromagnon-like excitation arising from the cycloidal spin fluctuation [39]. The sharp antiferromagnetic resonance (AFMR) with much smaller spectral intensity, which is active for the magnetic field component of light, is discerned at $2.5 \mathrm{meV}$ in the $A$-type phase [Fig. 4(a)]. In contrast, the development of two clear peaks, at 2.2 and $6.7 \mathrm{meV}$, is identified in the cycloidal-spin phase [Fig. 4(b)]. For the E-type phase [Fig. 4(c)], an electromagnon-like feature is also revealed in this study: three pronounced peaks appear at 2.9, 4.1 and $5.9 \mathrm{meV}$ that are in a frequency region typical of magnetic excitations.

Figure 5(a) shows the temperature dependence of Im $[\varepsilon \mu]$ spectra for $\mathrm{LuMnO}_{3}$. In paramagnetic phase $(90 \mathrm{~K})$, only the contribution from the low energy tail of the higher-lying phonon modes can be seen. The broad absorption band develops from slightly above $T_{N}=40 \mathrm{~K}$
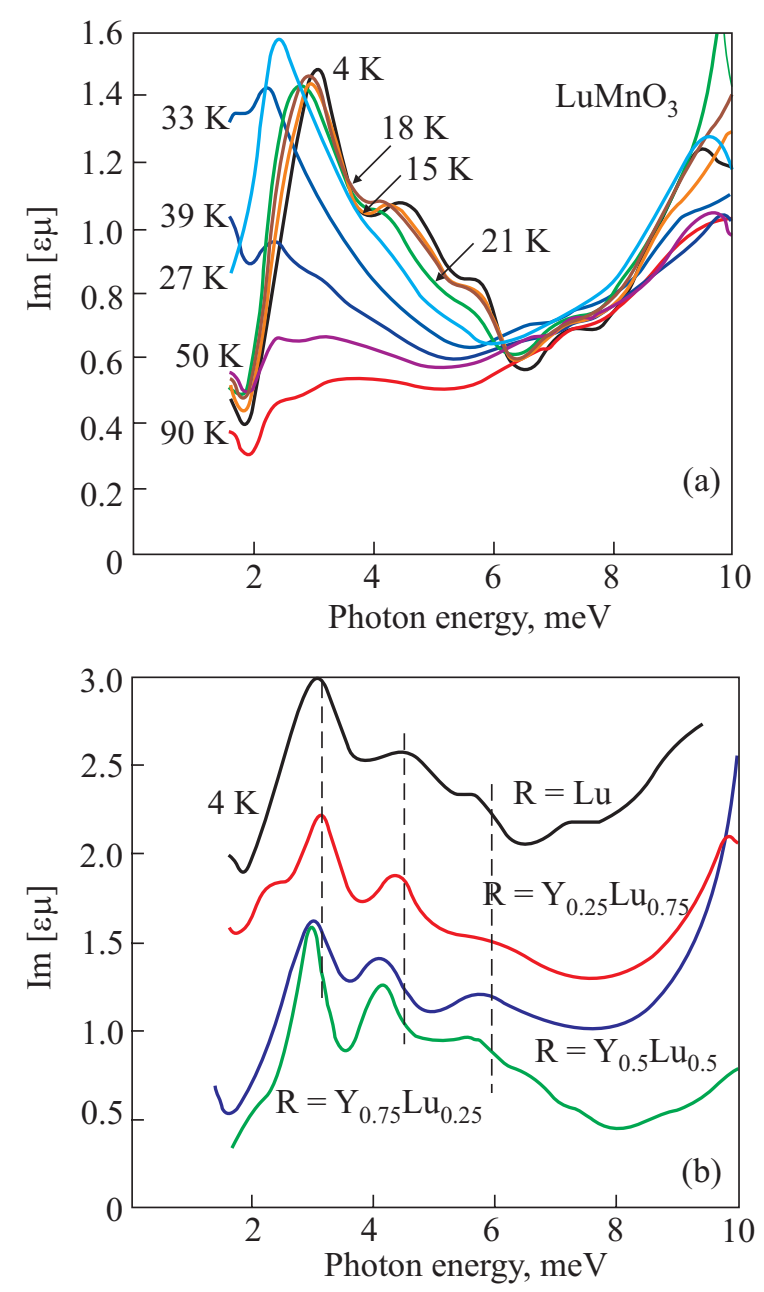

Fig. 5. (a) Temperature dependence of $\operatorname{Im}[\varepsilon \mu]$ spectra for $\mathrm{LuMnO}_{3}$; (b) $\mathrm{Im}[\varepsilon \mu]$ spectra of the $E$-type phase at $4 \mathrm{~K}$ for $\mathrm{Y}_{1-y} \mathrm{Lu}_{y} \mathrm{MnO}_{3}(y=0.25,0.5,0.75$ and 1.0) with vertical offset for clarity. Peak positions are indicated by the vertical dotted lines. (Reproduced from Ref. 40.) with decreasing temperature, and then it shows rapid enhancement below $T_{N}$. This material undergoes the transition from the collinear sinusoidal to the $E$-type phase at $T_{C}=35 \mathrm{~K}$, accompanied by the emergence of ferroelectricity. In accordance with this phase transition, the broad absorption band turns into peak structures at 2.9, 4.7 and $5.7 \mathrm{meV}$ with slight hardening of the frequencies while decreasing temperature. The spectra at the lowest temperature $T=4 \mathrm{~K}$ for the $E$-type phase are displayed for the $\mathrm{Y}_{1-y} \mathrm{Lu}_{y} \mathrm{MnO}_{3}$ samples in Fig. 5(b). All the spectra show the three-peak feature in common.

Thus, the latest data in the THz time-domain spectroscopy on electromagnons provide the strong evidence that the full spectra of electro-active magnons in $\mathrm{RMnO}_{3}$ consists of three perfectly resolved absorption peaks around 2.5-3, 4.5-5, and 5.5-6 meV, in contrast to a single broad $1-5 \mathrm{meV}$ peak, as it was reported by earlier measurements $[9,10,26,27]$. The low-lying electromagnon survives even in the collinear spin ordered phase and thus this electromagnon can not be ascribed as arising due to the DM interaction in multiferroics.

The models describing electric-dipole active excitations in perovskites manganites will be considered in the next subsection.

Among the multiferroic compounds, bismuth ferrite $\mathrm{BiFeO}_{3}$ is obviously one of the most interesting systems with both ferroelectric and magnetic transitions above room temperature: with a Néel temperature at $T_{N}=650 \mathrm{~K}$ and a ferroelectric transition temperature at $T_{C}=1100 \mathrm{~K}$ [56], exhibiting one of the largest polarization values, $\sim 100 \mu \mathrm{C} / \mathrm{m}^{2}$ [57]. Another interesting point is that when it undergoes an antiferromagnetic ordering at $650 \mathrm{~K}$ an incommensurate structure is formed with an extremely long period of $620 \AA$.

Electromagnons in $\mathrm{BiFeO}_{3}$ were for the first time unraveled by Cazayous et al. [45] and Singh et al. [46] by means of low-energy inelastic light scattering technique. The two intense one-magnon branches are detected at 18.2 and $26.6 \mathrm{~cm}^{-1}$. The authors considered these modes as not a pure spin wave but corresponding to magnetic excitations with electric dipole moment in and out of the cycloidal plane. Yet, a possible origin of the observed magnetic resonances is not uniquely identified. (There are actually three reasonable models for the data, see Ref. 46 for more details.)

The observation of electric-dipole active magnons in the $\mathrm{RMn}_{2} \mathrm{O}_{5}$ compounds including non-rare-earth metal $\mathrm{YMn}_{2} \mathrm{O}_{5}$ [48] are reported [47-49]. The electromagnons polarization selection rules provide evidence in favor of symmetric exchange coupling mechanism in this system.

$\mathrm{THz}$ spectroscopy also has been used to reveal the electromagnon excitation in the paraelectric $\uparrow \uparrow \downarrow \downarrow$ collinear magnetic phase of triangular lattice antiferromagnet $\mathrm{CuFe}_{1-x} \mathrm{Ga}_{x} \mathrm{O}_{2}$ [50]. Surprisingly, this mode was found to vanish in the ferroelectric helimagnetic phase. These facts 
prove that neither ferroelectricity nor noncollinear magnetism are necessary condition for the appearance of electromagnon excitation, while the existing theories on electromagnon have focused on noncollinear magnets like helimagnets. The anticorrelation between the noncollinear magnetism and the emergence of electromagnon excludes the exchange striction mechanism as the origin of dynamical magnetoelectric coupling. The electric activity of the magnon in this compound was attributed by the authors to the modulation of the $p-d$ hybridization at the spin-twisted excited state via the spin-orbit interaction. This discovery suggests that similar electromagnon modes should be observable in a broad variety of paraelectric collinear magnets.

Magnons and electromagnons in a four-sublattice $\mathrm{CuFeO}_{2}$ have been investigated by both $\mathrm{THz}$ time-domain spectroscopy [51] and by means of inelastic neutron scattering [52]. The authors demonstrated that only the highenergy mode generates the oscillating electric polarization, which can be coupled with an ac electric field.

\subsection{Theoretical considerations}

A common opinion at present is that a whole series of perovskite can show different multiferroic states, where two representative mechanisms, the symmetric exchange strictions and antisymmetric DM strictions, are effective. For the latter case, the noncommensurate cycloidal magnetic structure produces the macroscopic (spontaneous) polarization, $\quad \mathbf{P}_{A} \sim \mathbf{e}_{n m} \times\left(\mathbf{S}_{n} \times \mathbf{S}_{m}\right) \sim \propto[\mathbf{S}(\nabla \mathbf{S})-(\mathbf{S} \nabla) \mathbf{S}]$, parallel to the spiral plane (here spins $\mathbf{S}_{n}$ and $\mathbf{S}_{m}$ in points $\mathbf{n}$ and $\mathbf{m}$ should not be coupled by inversion) [16-18]. In contrast, the ferroelectricity in the $E$-type phase originates from the symmetric exchange term expressed as $\mathbf{P}_{S} \sim \pi_{n m}\left(\mathbf{S}_{n} \cdot \mathbf{S}_{m}\right)$. It is naturally to expect that both mechanisms cause the dynamical interplay between magnetic and electric excitations. However, the dynamical response of multiferroic systems was found to be more complex and there is no general consensus on this issue so far.

To analyze the results of theoretical investigations, one may start by describing the perovskites manganites $\mathrm{RMnO}_{3}$ within a frustrated Heisenberg model with the Hamiltonian of the form [35,58-60]

$$
\begin{aligned}
\mathcal{H}_{0} & =J_{1} \sum_{\text {n.n. }} \mathbf{S}_{n} \cdot \mathbf{S}_{m}+J_{2} \sum_{\text {n.n.n. }} \mathbf{S}_{n} \cdot \mathbf{S}_{m}+ \\
& +J_{c} \sum_{\text {n.n. }} \mathbf{S}_{n} \cdot \mathbf{S}_{m}+D_{\alpha} \sum_{n}\left(\mathrm{~S}_{n}^{\alpha}\right)^{2} .
\end{aligned}
$$

Here $J_{1}(<0)$ stands for the ferromagnetic interaction between the nearest-neighbor spins in the $a b$ plane; $J_{2}$ и $J_{c}$ (both $>0$ ) is the next-nearest-neighbor in this plane and interlayer interactions, respectively (see Fig. 1). As already mentioned, in this class of materials, the exchange interaction $J_{1}$ is very small relative to that of other perovskites compounds and the condition $J_{2} /\left|J_{1}\right|>0.5$ is typically fulfilled. Thus, the ground state is a cycloidal spin order with the spiral angle $\theta$ being equal to $\cos \theta=-J_{1} / 2 J_{2}$. A uniaxial anisotropy parameter $D_{\alpha}$ establishes the directions of the cycloidal plane. The evaluation of system parameters gives, for example, for $\mathrm{DyMnO}_{3}, \mathrm{TbMnO}_{3}$ and $\mathrm{Gd}_{0.7} \mathrm{~Tb}_{0.3} \mathrm{MnO}_{3}[35,58-60]$ that for all these compounds a noncommensurate magnetic structure is realized with the $c b$-cycloidal plane (i.e., $\alpha=a$ ) for $\mathrm{DyMnO}_{3}$ and $\mathrm{TbMnO}_{3}$, and $a b$-cycloidal plane (i.e., $\alpha=c$ ) for $\mathrm{Gd}_{0.7} \mathrm{~Tb}_{0.3} \mathrm{MnO}_{3}$.

When the external electric field $\mathbf{E}$ is applied, the spin Hamiltonian of the system can be written as $\mathcal{H}=\mathcal{H}_{0}-\mathbf{E} \cdot \mathbf{P}$, where $\mathcal{H}_{0}$ is a Hamiltonian of the frustrated Heisenberg model (1), and $\mathbf{P}$ is an electric dipole moment which depends on spin configuration. The electric dipole moment associated with a pair of spins on the nearest-neighbor bonds has been calculated within a microscopic theory [58-60]. It was found that the dominated symmetric spin depend component is given by

$$
\mathbf{P}_{S}=\sum_{\text {n.n. }} \pi_{n m}\left(\mathbf{S}_{n} \cdot \mathbf{S}_{m}\right), n \neq m .
$$

Here the vector $\pi_{n m}$ is nonzero if the inversion symmetry is absent at the center of $\mathbf{n}$ and $\mathbf{m}$ positions. For the cycloidal spin structure, the local symmetric spin polarization terms $\mathbf{P}_{S}$ are aligned antiferroelectrically [58-60]. Thus, static part of (2) is zero $\mathbf{P}_{S}=0$ and the symmetric term does not violate the fact that the total static ferroelectricity occurs solely due to the antisymmetric spin dependent term of the form: $\mathbf{P}_{A} \sim \mathbf{e}_{n m} \times\left(\mathbf{S}_{n} \times \mathbf{S}_{m}\right)$. But the dynamical fluctuations of $\mathbf{P}_{S}$ contribute to the electromagnon excitation. Especially, in the noncollinear ground state, the single-magnon processes at the zone edge originate from the term (2) (see Fig. 6).

The lower-lying magnetic excitations of the model (1), (2) are described by the liner spin wave approach. Using a standard procedure (see, e.g., [61]), the spin operator can be approximated by the Bose ones and then, by well known u-v-Bogolyubov transformation, diagonalized the Hamiltonian. In a spin wave (magnon) creation $\beta_{k}^{+}$and annihilation $\beta_{k}$ operators the polarization (2) is written as one-, two-, etc. magnon processes [35,58-60]:

$$
\begin{gathered}
\mathbf{P}_{S}=i S \sqrt{S N} \sin \theta \times \pi^{(1)}\left(\mathbf{k}_{2 \pi}\right)\left(\beta_{k_{2 \pi}}^{+}-\beta_{k_{2 \pi}}\right)- \\
-i S \sin (\theta / 2) \sum_{k} \pi^{(2)}(\mathbf{k})\left(\beta_{k}^{+} \beta_{-k-k_{2 \pi}}^{+}-\beta_{k} \beta_{-k-k_{2 \pi}}\right)+\ldots
\end{gathered}
$$

Here the vector $\pi^{(1)}$ has only a component parallel to the anisotropy axis $\alpha$. The second term in Eq. (3) corresponds to the absorption due to simultaneous two-magnon absorption process. The optically active two-magnon excitations are possible over the full magnon bandwidth with the contribution of $+k$ and $-k$ magnons, and hence should spread the spectral weight up to the energy twice of the magnon bandwidth. A possible effect of electric-dipole active twomagnon process on the observed characteristics is dis- 

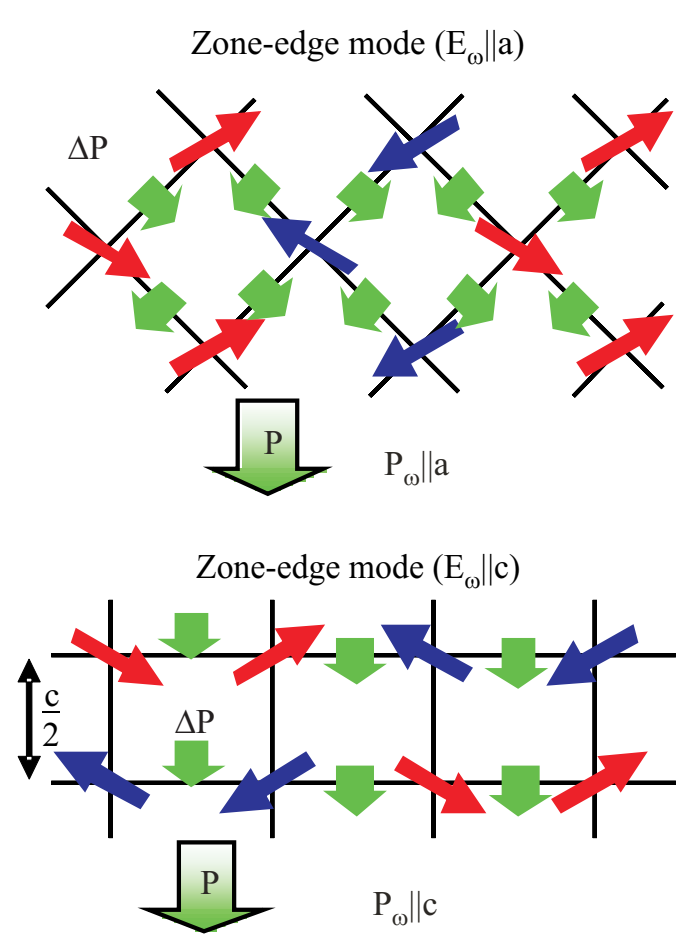

Fig. 6. The schematic zone-edge magnon in $a b$ and $b c$ planes. (Reproduced from Ref. 44.)

cussed in Ref. 30. In general, the effect of multi-magnon absorptions are negligible except near the Néel ordered state and, in the following, we focus on the first component in Eq. (3).

In the one-magnon process, spin waves with $\mathbf{k}_{2 \pi}=$ $=(2 \pi / a, 0,0), \mathbf{k}=(0,2 \pi / b, 0)$ and energy $\omega_{2 \pi}$ are induced. Introducing the electric polarization tensor, $P_{\alpha}(\omega)=$ $=\alpha_{\alpha \alpha \alpha}(\omega) E_{\alpha \omega}$, for the imaginary part of the tensor $\alpha_{\alpha \alpha}(\omega)$ the expression is obtained $[35,60]$

$$
\operatorname{Im} \alpha_{\alpha \alpha}^{(1)}(\omega)=N S^{2} \pi^{2}\left(k_{2 \pi}\right) \sin ^{2} \theta \delta\left(\omega-\omega_{2 \pi}\right) \delta_{\alpha, a} .
$$

The performed estimations yield that one-magnon absorption (4) is rather strong in a wide range of parameters except for near ferromagnetic and antiferromagnetic phases.

The approach (1), (2) perfectly reproduces the higher energy peak at $\sim 8 \mathrm{meV}$ for $\mathrm{TbMnO}_{3}$ and peak at $\sim 5 \mathrm{meV}$ for $\mathrm{DyMnO}_{3}[35,60]$. However, within this scenario the lower energy peak with substantial oscillator strength around $\sim 2 \mathrm{meV}$ is not obtained. One should remember that in general the full spectra of electro-active magnons in $\mathrm{RMnO}_{3}$ consist of three well manifested peaks of absorption around 2.5-3, 4.5-5, and 5.5-6 meV [44]. The inclusion of the antisymmetric term $\mathbf{P}_{A} \sim \mathbf{e}_{n m} \times\left(\mathbf{S}_{n} \times \mathbf{S}_{m}\right)$ does not improve the situation, because this interaction is nonzero in cycloidal spin phase and may be responsible for the one-magnon absorption only in geometry $\mathbf{E}_{\omega} \| a$. Thus, within the framework of the model (1), (2), the question about the origin of lower energy peak remains open.

Low-lying absorption can be obtained assuming that the spin configuration is subjected to the significant deviation from a pure cycloidal one with uniform spin rotation angle and contains higher harmonics [43]. The proposed model is based on the models (1), (2) but, in addition, contains the single-ion spin anisotropy within cycloid plane [i.e., in plane normal to axis $\alpha$ in Eq. (1)] and the biquadratic interaction between two neighbor spins in the $a b$ plane. The latter is given by

$$
\mathcal{H}_{\text {biq }}=-J_{\text {biq }} \sum_{\text {n.n. }}\left(\mathbf{S}_{n} \mathbf{S}_{m}\right)^{2} ; \mathbf{n}, \mathbf{m} \perp \alpha .
$$

These two additional terms cause a nonuniform spin rotation and effectively modulate a cycloid structure.

Within the approach [43], the calculated electromagnon spectrum was in a good agreement with experiment. Particularly, it was found that irrespective of spiral-plane orientation the absorption at low energy is enhanced strongly by weak anisotropy within cycloid plane or by the weak biquadratic interaction.

Yet, further investigations are necessary to examine the model [43]. In particular, as pointed in Ref. 38, the effects due to a magnetically induced incommensurate oscillatory polarization can be also important. A further generalization of the approach [43] which includes the spin-phonon coupling has been considered in Ref. 62. Considering the lattice degree of freedom the authors succeeded in reproducing the entire phase diagram of $\mathrm{RMnO}_{3}$ for the first time and explained several experimental puzzles. However, the dynamical properties of the systems are not discussed in Ref. 62.

One of a key test for any theoretical model will be its ability to reproduce a sensitivity of the electric-dipole active magnon spectra to the rare-earth ionic radius observed in $\mathrm{RMnO}_{3}$ multiferroics.

The physical picture proposed in Refs. 35, 43, 58-60, 62 reveals an important condition for a search of new class of materials. Namely, in dynamics the effect of symmetric, $\sim \mathbf{S} \cdot \mathbf{S}$, magnetostriction can be larger that the usually expected antisymmetric, $\sim \mathbf{S} \times \mathbf{S}$, contribution. This enables the researchers to search for the electric-dipole active magnetic excitations not only in multiferroics but in compounds without static electric polarization, as well. The necessary condition is only the absence of coupling of magnetic ions positions by inversion symmetry. A few examples of such multi-sublattice magnets with collinear magnetic order are discussed in the next section.

\section{Electric-dipole active exchange modes in collinear magnets}

The above-considered systems are antiferromagnets with noncommensurate magnetic structure. In such a case, the appearance of electric dipole moment is due to disappearance of a centre of inversion in the ordered phase and direct coupling between magnetic and electric order parameters. There is a limited number of compounds where the 
both conditions are satisfied. Overwhelming majority of magnetic compounds contain a few magnetic ions in primitive magnetic cell and are ordered into commensurate and (in exchange approximation) collinear magnetic phase. In this case, the spectrum of proper magnetic excitation contains acoustic modes (AMs), with in-phase oscillation of spins within a magnetic elementary cell, as well as modes similar to optic phonons, i.e., with antiphase oscillation of spins in an elementary cell. Frequency of such oscillations, which are called the exchange modes (EMs), is of the order of exchange interaction energy and, in typical cases, pertain to $\mathrm{THz}$ and even optic frequency range. Excitation of EMs by a magnetic component of light is possible only if the former is magnetically-active, i.e., is coupled to oscillations of the ferromagnetic vector $\mathbf{M}$ of the system. However, the coupling of EM and $\mathbf{M}$ is due to relativistic and exchange-relativistic interactions, and therefore the intensity of absorption by EM is weak compared to AM. For nonmagnetic-active EMs, i.e., for those of EMs which are not coupled with oscillations of the ferromagnetic vector $\mathbf{M}$, the interaction with magnetic field is impossible by virtue of selection rules, for example, in the case of EMs which are odd under inversion. The natural way to resonantly excite magnetic-system EM which is odd under inversion, is to expose on them to a high-frequency electric field.

The presence of electrically-active EMs in a multisublattice magnet with magnetic ions located in noncentrosymmetric positions and appearance of an antiferroelectric resonance (AFER), were theoretically predicted in Refs. 63-70, while some remarks concerning such a possibility can be found even in earlier publications [71,72]. (We keep here the term «antiferroelectric resonance» because the electric-dipole active EM is oscillation of the antiferromagnetic vectors $\mathbf{L}$ and to distinguish this type of oscillations from electromagnons in multiferroics.) It is significant that in AFER the electric component of light is directly connected with the vectors $\mathbf{L}$. This is a main distinction to the case of multiferroics.

\subsection{General conditions for the existence of AFER}

Let us formulate the general conditions for electricdipole active magnetic oscillations in magnetic structures whose ground state in the exchange approximation is collinear [64]. As is known, for symmetry analysis it is convenient to introduce linear combinations of sublattice magnetizations $\mathbf{M}_{\zeta}(\zeta=1,2, \ldots, n)$; that are the ferromagnetism $\mathbf{M}$ and antiferromagnetism $\mathbf{L}_{v}(v=1,2, \ldots n-1)$ vectors. In the general case the EM and AM are of electric-dipole type if the dynamic compounds of the antiferromagnetism and ferromagnetism vectors which correspond to them transform just like the compounds of the electric polarization vector $\mathbf{P}$. More specifically, in centrosymmetric crystals it is necessary to distinguish between structures which are even and odd under inversion.
3.1.1. The structure $\overline{1}(+)$. If a system whose structure is even under inversion contains magnetic ions which are not located at inversion centers, then in addition to the basic even antiferromagnetism vector $\mathbf{L}_{0}$, there exists at least one other antiferromagnetism vector $\boldsymbol{\ell}$ which is odd under inversion. In the thermodynamic potential of such a magnet it is possible to have invariants of the form

$$
K_{\alpha \beta \gamma} E_{\alpha} L_{0 \beta} l_{\gamma}, \quad \alpha, \beta, \gamma=x, y, z ; \quad K_{\alpha \beta \gamma}=\text { const. }
$$

In a static electric field, Eq. (6) leads to the appearance of $\ell_{\gamma} \sim E_{\alpha}$. In analogy with the magnetoelectric effect this phenomenon can be called antiferroelectric effect (AFEE). In an ac electric field $\mathbf{E} \sim \mathbf{E}_{\omega} \exp (\mathrm{i} \omega \mathrm{t})$, the relation (6) causes oscillation of the vector $\boldsymbol{\ell}$, and for $\omega=\omega_{0}\left(\omega_{0}\right.$ is the exchange frequency) excitation of EM - an antiferroelectric resonance. Such type of homogeneous excitation is always decoupled with even under inversion excitations. This specific of dynamics does not depend on external magnetic field orientation and is common for all magnetic crystals with even spin structure.

A direct indication of the possibility of experimental observation of AFER is provided by experiments in which an ac electric field induces transitions between magnetic levels of paramagnetic ions in noncentrosymmetric sites, i.e., transitions from a state $(l, m)$ to states $(l, m+1)$ where $l$ is the orbital and $m$ the magnetic quantum number. Such transitions were observed in [2] (see also Refs. 3-5). As we have shown here, in centrosymmetric crystals which have high concentrations of magnetic ions and magnetic structures which are even under inversion (e.g., of $\alpha-\mathrm{Fe}_{2} \mathrm{O}_{3}$ type) one consequence of these transitions will be magnetic excitations of exchange type. Thus, the physics of AFER combines the physical mechanisms of electric-dipole active paramagnetic resonance associated with impurity magnetic ions in noncentrosymmetric position [3,73], and of absorption and scattering of light in magneticallyordered crystals [74-76]. In systems with magnetic ions occupying centers of inversion, the electric dipole activity of the magnetic modes can be due to, e.g., the additional effect of a constant electric field.

3.1.2. The structure $\overline{1}(-)$. For structures odd under inversion, it is necessary to distinguish between two cases according to parity relative to a translation $\tau$.

i) Systems with $\overline{1}(-), \boldsymbol{\tau}(+)$ pertain to antiferromagnets whose thermodynamic potentials include an invariant of the form

$$
K_{\alpha \beta \gamma} E_{\gamma} L_{0 \alpha} m_{\beta}
$$

where $m_{\beta}$ is the magnetic moment of the system. If in this case also exists an antiferromagnetic vector $\boldsymbol{\ell}$ which is even under inversion, then it is also possible to have an invariant of the form (6). This invariant gives rise to the presence of AFER and AFEE, while (7) makes possible excitation of AM by an electric field, i.e., electromagnons. As far as an 
invariant $\sim \boldsymbol{\ell}_{\alpha} \mathrm{M}_{\beta}$ is also possible, AM and EM will be excited by both magnetic and electric fields of light.

ii) In systems with $\overline{1}(-), \tau(-)$ (the magnetic unit cell is larger than the crystallographic unit cell) the invariant (7) is forbidden and there is no static magnetoelectric effect. Nevertheless, an invariant of the form (6) is possible, where now the antiferromagnetic vector $\boldsymbol{l}$ is odd under translation and even under inversion. In this system there will be both AFEE and AFER. However, in this magnetic phase the electric-dipole active and magnetic-dipole-active spin excitations are uncoupled in dynamics.

The essential features of AFER in noncentrosymmetric crystals will be illustrated with two examples: hematite $\left(\alpha-\mathrm{Fe}_{2} \mathrm{O}_{3}, T_{N}=948 \mathrm{~K}\right)$ and chromium oxide $\left(\mathrm{Cr}_{2} \mathrm{O}_{3}\right.$, $\left.T_{N}=307 \mathrm{~K}\right)$, where the former possesses even under inversion magnetic order, while the magnetic structure of the latter is odd.

\subsection{AFER in $\alpha-\mathrm{Fe}_{2} \mathrm{O}_{3}$ and $\mathrm{Cr}_{2} \mathrm{O}_{3}$}

Hematite and chromium (III) oxide are well known four-sublattice rhombohedral antiferromagnets with the crystallographic group $R \overline{3} c \equiv D_{3 d}^{6}$. Magnetic ions are in fourfold position $4 c\left\{3_{z}\right\}$. We introduce the following linear combinations of the sublattice magnetizations $\mathbf{M}_{\zeta}$ :

$$
\begin{aligned}
& \mathbf{M}=\mathbf{M}_{1}+\mathbf{M}_{2}+\mathbf{M}_{3}+\mathbf{M}_{4}=4 \mathbf{M}_{0} \mathbf{m}, \\
& \mathbf{L}_{1}=\mathbf{M}_{1}-\mathbf{M}_{2}-\mathbf{M}_{3}+\mathbf{M}_{4}=4 \mathrm{M}_{0} \boldsymbol{\ell}_{1}, \\
& \mathbf{L}_{2}=\mathbf{M}_{1}-\mathbf{M}_{2}+\mathbf{M}_{3}-\mathbf{M}_{4}=4 \mathrm{M}_{0} \boldsymbol{\ell}_{2}, \\
& \mathbf{L}_{3}=\mathbf{M}_{1}+\mathbf{M}_{2}-\mathbf{M}_{3}-\mathbf{M}_{4}=4 \mathrm{M}_{0} \boldsymbol{\ell}_{3},
\end{aligned}
$$

where $\mathrm{M}_{0}$ is the magnitude of the sublattice magnetization.

i) For $\alpha-\mathrm{Fe}_{2} \mathrm{O}_{3}$, the classification of the vectors (9) relative to the irreducible representations of the group $D_{3 d}^{6}$ is shown in Table 1 . The vectors $\mathbf{m}$ and $\boldsymbol{\ell}_{1}$ generate even, and $\boldsymbol{\ell}_{2}$ and $\boldsymbol{\ell}_{3}$, odd, representations of this group. Using the table, it is easy to obtain the spin system Hamiltonian $\mathcal{H}_{0}$ (see, e.g., Refs. 63, 64, 77). In linear approximation, the vectors (9) homogeneous oscillations correspond to two AMs and two EMs. The AMs are due to oscillation of even regarding to inversion vectors $\mathbf{m}$ and $\boldsymbol{\ell}_{1}$, and are coupled with magnetic light component, i.e., are magneticallyactive oscillations. In contrast, the EMs are due to odd regarding to inversion vectors, $\boldsymbol{\ell}_{2}$ and $\boldsymbol{\ell}_{3}$, oscillation of and thus cannot be coupled with magnetic light component, as well as with the AMs. On the other hand, electric field could be coupled with the vectors $\boldsymbol{\ell}_{2}$ and $\boldsymbol{\ell}_{3}$ (see Table 1), i.e., EMs are electric-dipole active excitations. These properties of the AMs and EMs preserve both in easy-axis and easy-plane phases.

When electric field $\mathbf{E}$ is applied the system Hamiltonian is given by $\mathcal{H}=\mathcal{H}_{0}-\mathbf{E} \cdot \mathbf{P}$, where the additional component $\mathbf{E} \cdot \mathbf{P}$ is expressed as $[63,64]$
Table I. Magnetic configurations which are irreducible relative to the crystallographic group $D_{3 d}^{6}$.

\begin{tabular}{c|c|c}
\hline \hline $\begin{array}{c}\text { Irreducible } \\
\text { representation }\end{array}$ & $\begin{array}{c}\text { Irreducible } \\
\text { spin configurations }\end{array}$ & $\begin{array}{c}\text { Polarization of ac } \\
\text { electric and mag- } \\
\text { netic fields }\end{array}$ \\
\hline \hline$A_{1 g}$ & $\ell_{1 z}$ & - \\
$A_{2 g}$ & $m_{z}$ & $H_{z}$ \\
$E_{g}$ & $\left(m_{+}, m_{-}\right),\left(\ell_{1}+\ell_{2-}\right)$ & $\left(H_{+}, H_{-}\right)$ \\
$A_{1 u}$ & $\ell_{3 z}$ & - \\
$A_{2 u}$ & $\ell_{2 z}$ & $E_{z}$ \\
$E_{u}$ & $\left(\ell_{2+}, \ell_{2-}\right),\left(\ell_{3+},-\ell_{3-}\right)$ & $\left(E_{+}, E_{-}\right)$ \\
\hline \hline
\end{tabular}

$$
\begin{aligned}
& \left(2 M_{0}\right)^{-1} \mathbf{E} \cdot \mathbf{P}=E_{x}\left\{R_{3}\left(\ell_{1 z} \ell_{3 y}-\ell_{1 y} \ell_{3 z}\right)+\right. \\
+ & \left.R_{2}\left(\ell_{1 z} \ell_{2 x}-\ell_{1 x} \ell_{2 z}\right)+r_{3}\left(m_{x} \ell_{3 z}-m_{z} \ell_{3 x}\right)\right\}+ \\
+ & E_{y}\left\{R_{3}\left(\ell_{1 x} \ell_{3 z}-\ell_{1 z} \ell_{3 x}\right)+R_{2}\left(\ell_{1 z} \ell_{2 y}-\ell_{1 y} \ell_{2 z}\right)+\right. \\
+ & \left.r_{3}\left(m_{y} \ell_{3 z}-m_{z} \ell_{3 y}\right)\right\}+E_{z}\left\{\pi_{1} \mathbf{m} \ell_{3}+\pi_{2} \ell_{1} \ell_{2}+\right. \\
+ & \left.R_{3 z}\left(\ell_{1 x} \ell_{3 y}-\ell_{1 y} \ell_{3 x}\right)+r_{2 z}\left(m_{x} \ell_{2 y}-m_{y} \ell_{2 x}\right)\right\} .
\end{aligned}
$$

Here the antiferroelectric constants $\pi_{i}$ have their origin in exchange, while $R_{i}$ and $r_{i}$ are due to relativistic-exchange effects; terms of relativistic origin are neglected. The microscopy origin of the invariants $\sim\left(\pi_{1} \mathbf{m} \cdot \ell_{3}+\pi_{2} \boldsymbol{\ell}_{1} \cdot \ell_{2}\right)$ is due to the symmetric exchange strictions, Eq. (2), i.e., the electric-dipole originates from dynamic polarization of electronic bonds of two magnetic ions not connected by inversion. The generation of other terms is provided by the antisymmetric DM interaction $\sim \mathbf{e}_{n m} \times\left(\mathbf{S}_{n} \times \mathbf{S}_{m}\right)$.

Thus, for the easy-plane phase $\left(\ell_{1 y}=1, \mathbf{H} \| x\right)$ we have, to linear approximation in the spin deviations, for the components of the electric polarization vector [64]:

$$
\begin{gathered}
P_{x}=-4 M_{0}\left(R_{3}-r_{3} m\right) \ell_{3 z}, \quad P_{y}=4 M_{0} R_{2} \ell_{2 z}, \\
P_{z}=4 M_{0}\left[\left(\pi_{2}+r_{2 z} m\right)+\left(\pi_{1} m-R_{3 z}\right) \ell_{3 z}\right] .
\end{gathered}
$$

Introducing the electric polarization tensor, $P_{i}(\omega)=$ $=\alpha_{i j}(\omega) E_{j \omega}$, we find that the spin part of $\alpha_{i j}(\omega)$ has the following nonzero components:

$$
\begin{gathered}
\alpha_{x x}(\omega)=-4 M_{0} \gamma^{2} R_{3}^{2}\left(H_{e 1}+H_{e 2}\right)\left(\omega^{2}-\omega_{01}^{2}\right)^{-1}, \\
\alpha_{y y}(\omega)=-4 M_{0} \gamma^{2} R_{2}^{2}\left(H_{e 1}+H_{e 3}\right)\left(\omega^{2}-\omega_{02}^{2}\right)^{-1}, \\
\alpha_{y z}(\omega)=-\alpha_{z y}(\omega)=i 4 M_{0} \gamma^{2} R_{2}\left[R_{3 z}+\left(\pi_{2}-\pi_{1}\right) m\right] \omega \times \\
\times\left(\omega^{2}-\omega_{02}^{2}\right)^{-1}, \\
\alpha_{z z}(\omega)=4 M_{0} \gamma^{2}\left[R_{3 z}+\left(\pi_{2}-\pi_{1}\right) m\right]\left(\pi_{1} m-R_{3 z}\right) \times \\
\times\left(H_{e 1}+H_{e 2}\right)\left(\omega^{2}-\omega_{02}^{2}\right)^{-1} .
\end{gathered}
$$

Here $H_{e v}$ is the effective fields of exchange interactions; the explicit expressions for EMs $\omega_{01} / \gamma$ and $\omega_{02} / \gamma$ are given in Refs. 63, 64 ( $\gamma$ stands for gyromagnetic ratio). 
Similar calculations for the easy-axis phase $\left(\ell_{1 z}=1\right.$, $\mathbf{H} \| z)$ yield: $P_{x}=4 M_{0}\left(R_{3} \ell_{3 z}+R_{2} \ell_{2 x}\right), P_{y}=4 M_{0} R_{2} \ell_{2 y}$, and $P_{z}=4 M_{0} \pi_{2} \ell_{2 z}$. Calculating the oscillation amplitudes of the antiferromagnetic vectors $\boldsymbol{\ell}_{2}(t)$ and $\boldsymbol{\ell}_{3}(t)$ under the action of electric field of light, one obtains the following nonzero spin contributions to the electric tensor $\alpha_{i j}(\omega)$ :

$$
\begin{gathered}
\alpha_{x x}(\omega)=\alpha_{y y}(\omega)=4 M_{0} \gamma^{2}\left\{R_{3}^{2}\left(H_{e 1}+H_{e 2}\right)+\right. \\
\left.+R_{2}^{2}\left(H_{e 1}+H_{e 3}\right)\right\} \times \\
\times\left(\omega_{01} \omega_{02}-\omega^{2}\right)\left(\omega^{2}-\omega_{01}^{2}\right)^{-1}\left(\omega^{2}-\omega_{02}^{2}\right)^{-1} \\
\alpha_{x y}(\omega)=-\alpha_{y x}(\omega)=i 4 M_{0} \gamma^{2}\left\{R_{3}^{2}\left(H_{e 1}+H_{e 2}\right)+\right. \\
\left.+R_{2}^{2}\left(H_{e 2}+H_{e 3}\right)\right\} \times \\
\times 2 \omega H\left(\omega^{2}-\omega_{01}^{2}\right)^{-1}\left(\omega^{2}-\omega_{02}^{2}\right)^{-1}
\end{gathered}
$$

The explicit expressions for EM $\omega_{01} / \gamma$ and $\omega_{02} / \gamma$ in this phase are given in Refs. 63, 64. Thus in hematite the absorption of electric component of light on EMs is enhanced by the exchange fields $H_{e v}$.

The magnitude of antiferroelectric constants for $\alpha-\mathrm{Fe}_{2} \mathrm{O}_{3}$ can be estimated from experiments on the shift of the paramagnetic resonance lines for $\mathrm{Fe}^{3+}$ ions [3]. The latter give a value of $\sim 10^{-2}$ for the single-ion spin-Hamiltonian constant. In systems with a high concentration of magnetic ions, contributions to the magnetoelectric effects come also from ion-ion interactions (in particular from exchange and relativistic-exchange interactions), which in individual cases increase the value of the constant by an order of magnitude [78]. Therefore in $\alpha-\mathrm{Fe}_{2} \mathrm{O}_{3}$ (apparently) $R_{i} \sim 10^{-1}$. For the value of $\pi$ it is reasonable to expect an order of magnitude larger. It should also be noted that the contribution from the exchange origin invariants is proportional to the magnetization; therefore, in the canted phases their effect can significantly exceed those from the exchangerelativistic invariants.

(ii) The crystal $\mathrm{Cr}_{2} \mathrm{O}_{3}$ possesses a magnetic structure which is odd under inversion [77]. The magnetic class of $\mathrm{Cr}_{2} \mathrm{O}_{3}$ includes inversion combines with the time-reversal operation $\overline{I R}$, and this result in a linear magnetoelectric effect [79-81]. The ground state is $\boldsymbol{\ell}_{3} \| \mathrm{z}$. In the exchange approximation the acoustic type of oscillations correspond to transverse oscillations of the vectors $\ell_{3}$ and $\mathbf{m}$, while those of exchange type correspond to transverse oscillation of the vectors $\boldsymbol{\ell}_{1}$ and $\boldsymbol{\ell}_{2}$. Due to the Dzyaloshinksii interaction, $H_{D}$, the modes are dynamically coupled. Without pausing for detailed calculations we will present the final results [64]. In linear approximation, for the electric polarization we have: $P_{x}=4 M_{0}\left(r_{3} m_{x}-R_{3} \ell_{1 y}\right)$ and $P_{y}=4 M_{0} \times$ $\times\left(r_{3} m_{y}+R_{3} \ell_{1 x}\right)$. The nonzero spin contribution to the electric polarization tensor at AMs, $\omega_{A 1} / \gamma=\varepsilon_{\mathrm{A}}+H$ and $\omega_{A 2} / \gamma=\varepsilon_{A}-H$, is described by the expressions

$$
\begin{gathered}
\alpha_{x x}(\omega)=\alpha_{y y}(\omega)=4 M_{0} \gamma^{2} r_{3}^{2}\left\{H_{A 3}-H_{D}^{2}\left(H_{e 2}+H_{e 3}\right)^{-1}\right\} \times \\
\times\left(\omega^{2}-\varepsilon_{A}^{2}+H^{2}\right)\left(\omega^{2}-\omega_{A 1}^{2}\right)^{-1}\left(\omega^{2}-\omega_{A 2}^{2}\right)^{-1},
\end{gathered}
$$

$$
\begin{aligned}
& \alpha_{x y}(\omega)=- \alpha_{x y}(\omega)=i 4 M_{0} \gamma^{2} \mathrm{r}_{3}^{2}\left\{H_{A 3}-H_{D}^{2}\left(H_{e 2}+H_{e 3}\right)^{-1}\right\} \times \\
& \times \omega H\left(\omega^{2}-\omega_{A 1}^{2}\right)^{-1}\left(\omega^{2}-\omega_{A 2}^{2}\right)^{-1} .
\end{aligned}
$$

Within the same accuracy, the $\alpha_{i j}(\omega)$ nonzero component structure at EMs, $\omega_{01} / \gamma=\varepsilon_{0}+H$ and $\omega_{02} / \gamma=\varepsilon_{0}-H$, has the form

$$
\begin{gathered}
\alpha_{x x}(\omega)=\alpha_{y y}(\omega)=4 M_{0} \gamma^{2} R_{3}^{2}\left(H_{e 2}+H_{e 3}\right) \times \\
\times\left(\omega^{2}-\varepsilon_{0}^{2}+H^{2}\right)\left(\omega^{2}-\omega_{01}^{2}\right)^{-1}\left(\omega^{2}-\omega_{02}^{2}\right)^{-1}, \\
\alpha_{x y}(\omega)=-\alpha_{y x}(\omega)=-i 4 M_{0} \gamma^{2} R_{3}^{2}\left(H_{e 2}+H_{e 3}\right) \times \\
\times \omega H\left(\omega^{2}-\omega_{01}^{2}\right)^{-1}\left(\omega^{2}-\omega_{02}^{2}\right)^{-1} .
\end{gathered}
$$

The explicit expressions for EM and EM at zero magnetic field, $\varepsilon_{A}$ and $\varepsilon_{0}$, are given in Ref. 64 .

Since the vectors $\left(\boldsymbol{\ell}_{1}, \mathbf{m}\right)$, which are even under inversion, and the vectors $\left(\boldsymbol{\ell}_{2}, \ell_{3}\right)$, which are odd under inversion, participate in the EM and AM oscillations, these modes can be excited both by magnetic and electric components of light. However, from an experimental point of view the important is the magnitude of the absorption by the EM and AM. The estimations of the magnetic $\chi_{i j}(\omega)$ and electric $\alpha_{i j}(\omega)$ susceptibility near the resonance frequency yield: $\alpha\left(\omega_{0}\right) \sim$ $\sim \gamma M_{0} R^{2}\left(\omega-\omega_{0}\right)^{-1}, \quad \chi\left(\omega_{0}\right) \sim \gamma M_{0}\left(H_{A} / H_{e}\right)\left(\omega-\omega_{0}\right)^{-1}$ for $\mathrm{EM}$, and $\alpha\left(\omega_{A}\right) \sim \gamma M_{0} R^{2}\left(H_{A} / H_{e}\right)^{1 / 2}\left(\omega-\omega_{A}\right)^{-1}, \chi\left(\omega_{A}\right) \sim$ $\sim \gamma M_{0}\left(H_{A} / H_{e}\right)^{1 / 2}\left(\omega-\omega_{A}\right)^{-1}$ for AM. Thus, the intensity of the absorption an electric field of light by EM is $\alpha\left(\omega_{0}\right) / \alpha\left(\omega_{A}\right) \sim\left(H_{A} / H_{e}\right)^{1 / 2}$ times large that the absorption by AM. For magnetic field of light the situation is reverse: $\chi\left(\omega_{0}\right) / \chi\left(\omega_{A}\right) \sim\left(H_{A} / H_{e}\right)^{1 / 2}$. It is also clear that for $R^{2}>$ $>H_{A} / H_{e}$, the excitation of EM by electric field of light is easier than by magnetic field.

For quantitative estimates of the antiferroelectric interaction constants in $\mathrm{Cr}_{2} \mathrm{O}_{3}$ we make use of the results of experimental and theoretical studies of the magnetoelectric effect in this compound $[78,82]$. The latter give for the parameters of the spin Hamiltonian the value $\pi \approx 0.5, R \approx 0.05$. Taking into account that $H_{A} / H_{e} \sim 10^{-4}$ in $\mathrm{Cr}_{2} \mathrm{O}_{3}$, the conditions for detection of EM as peak in $\varepsilon(\omega)$ is more favorable than those in $\mu(\omega)$.

The EM frequencies of $\alpha-\mathrm{Fe}_{2} \mathrm{O}_{3}$ and $\mathrm{Cr}_{2} \mathrm{O}_{3}$ are located in the infrared wavelength band. Therefore the features of experimental observation of AFER in these compounds are close to those at $\mathrm{THz}$ frequencies [15] or optics [75,76]. If the dimensions of the sample are comparable with or smaller than the wavelength of the ac electric field, then the results above is directly applicable. For bulk samples of $\alpha-\mathrm{Fe}_{2} \mathrm{O}_{3}$ and $\mathrm{Cr}_{2} \mathrm{O}_{3}$, the equations of motion for the vectors (9) must be considered jointly with the Maxwell equations. In this case, the magnetooptic effects will be restructured. In particular, near the exchange resonances it is possible to resonantly enhance such magnetooptic effects as Faraday rotation, the Cotton-Mouton effect, and the Kerr effect. A detailed analysis of these questions is outside the framework of the present report. The reader is referred to Refs. 66-70. 


\section{Electric-dipole active magnetic resonances in ferrimagnets}

The above considered systems are antiferromagnets, i.e., without spontaneous ferromagnetic moment. However, the most interesting materials for both future applications as well as the quest for fundamental principles are those where ferromagnetic magnetization and ferroelectric polarization are coexisting. A sole to date such type of compound where electromagnons are experimentally revealed is hexaferrite $\mathrm{Ba}_{2} \mathrm{Mg}_{2} \mathrm{Fe}_{12} \mathrm{O}_{22}[83,84]$.

As is known [85], at room temperature magnetic structure of $\mathrm{Ba}_{2} \mathrm{Mg}_{2} \mathrm{Fe}_{12} \mathrm{O}_{22}$ can be considered as two antiferromagnetically ordered sublattices $L$ and $S$. Below $195 \mathrm{~K}$ this structure transforms into the proper spiral with spiral axis [001]. Then, below $50 \mathrm{~K}$, the ordered conical-spin phase appears with finite conical angle $\theta$ for each sublattices. Kida et al. [83] observed a noticeable optical anisotropy in the $\mathrm{THz}$ spectra for the ordered conical-spin phase. The results obtained are presented in Figs. 7(a) and 7 (b) where, respectively, the real $\left(\varepsilon_{1}\right)$ and imaginary $\left(\varepsilon_{2}\right)$ parts of the complex dielectric permittivity at $5 \mathrm{~K}$ are shown for different polarization configurations. A gigantic

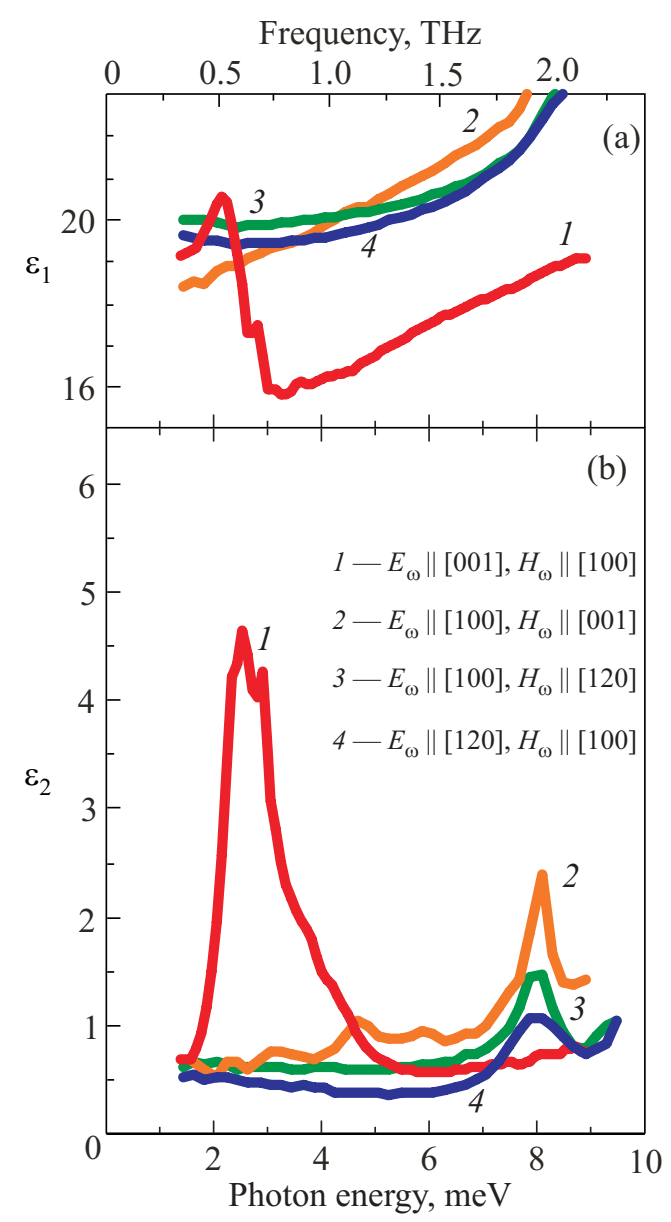

Fig. 7. Electric-dipole active magnetic resonance in $\mathrm{Ba}_{2} \mathrm{Mg}_{2} \mathrm{Fe}_{12} \mathrm{O}_{22}$. Real $\varepsilon_{1}$ (a) and imaginary $\varepsilon_{2}$ (b) parts of the complex dielectric constant spectra $\varepsilon(\omega)$ measured in the ordered conical spin phase; $T=5 \mathrm{~K}, H=0$. (Reproduced from Refs. 83, 84.) sharp resonance around $2.8 \mathrm{meV}$ in $\varepsilon_{2}(\omega)$ and a dispersive structure in $\varepsilon_{1}(\omega)$ are clearly seen, when electric and magnetic fields polarizations of the light were set parallel to $\mathbf{E}_{\omega} \|[001]$ and $\mathbf{H}_{\omega} \|$ [100]. Based on the complementary measurements of the magnon dispersion (the inelastic neutron scattering spectra and temperature dependence of the complex dielectric constant) the authors [84] firmly identified that the observed electric-dipole active mode around $2.8 \mathrm{meV}$ is magnetic in origin. In the configuration $\mathbf{E}_{\omega} \|$ [120] and $\mathbf{H}_{\omega} \|$ [100] another resonance can be discerned around $8 \mathrm{meV}$ [Fig. 7(b)]. The magnitude of this resonance is much smaller than that of $2.8 \mathrm{meV}$. The mode at $8 \mathrm{meV}$ disappears for $\mathbf{E}_{\omega} \|$ [001]. Therefore, this mode can be assigned also to an electric-dipole active mode, while allowed only for the in-plane $E_{\omega}$.

It was demonstrated $[83,84]$ that both longitudinal and transverse conical-spin structures can give rise to electricdipole active magnetic resonance, irrespective of the presence or absence of spontaneous electric polarization. Therefore, contrary to the origin of the ferroelectricity, i.e., the antisymmetric DM interaction $\left(\mathbf{S}_{n} \times \mathbf{S}_{m}\right)$, the symmetric exchange interaction (2) acting along [001] is likely the dominant source of the observed electromagnons in this hexaferrite.

Obviously, an interesting system is rare-earth garnets with the lattice of $\mathrm{Y}_{3} \mathrm{Fe}_{5} \mathrm{O}_{12}$. For compound $\mathrm{Dy}_{3} \mathrm{Fe}_{5} \mathrm{O}_{12}$, the attempt to find electromagnons was performed recently in Ref. 86. Far-infrared spectra of magneto-dielectric $\mathrm{Dy}_{3} \mathrm{Fe}_{5} \mathrm{O}_{12}$ garnet were studied between 13 and $100 \mathrm{~cm}^{-1}$ and at low temperatures between 5 and $80 \mathrm{~K}$. A combination of transmission, reflectivity, and rotating analyzer ellipsometry was used to identify the type of the dipole activity of the infrared modes. In addition to purely dielectric and magnetic modes, the authors observed several hybrid modes with a mixed magnetic and electric dipole activity. Microscopically, these modes can originate from the superexchange interaction between magnetic moments of Fe and Dy ions. It was speculated that the proximity of the Dy ${ }^{3+}$ exchange resonances to the frequency of the lowest optical phonon, local electric polarization, and the noncollinear spin structure for the Dy-Fe magnetic system are responsible for the mode hybridization. Further experimental and theoretical investigations are needed to understand unambiguously these results.

Among the compounds with garnet lattice a special place take those where magnetic ions occupy only rare-earth positions $\{c\}$. Examples of such garnets are $\mathrm{Tb}_{3} \mathrm{Ga}_{5} \mathrm{O}_{12}$ and $\mathrm{Dy}_{3} \mathrm{Al}_{5} \mathrm{O}_{12}$. At low temperature $(T<1 \mathrm{~K})$ the magnetic moments of rare-earth ions ordered forming noncollinear magnetic structure called the "kagome structure", when local easy-axis (and the spins of ions) are directed along three mutually orthogonal directions. Magnetic-strictions properties of such garnet were considered in [87]. Investigation the dynamical properties of these interesting systems is just in the beginning [88-90]. Recent advance in 
THz time-domain spectroscopy may stimulate the investigations on a variety of kagome spin structure magnets to uncover the electric-dipole active magnetic resonances in these compounds.

Just recently a new ferromagnetic ferroelectric $\mathrm{Mn}_{2} \mathrm{GeO}_{4}$ has been revealed in experiments [91]. The compound is shown to feature both a ferroelectric polarization and a ferromagnetic magnetization that are directly coupled and point along the same direction. The authors showed that a spin spiral generates ferroelectricity, and a canted commensurate order leads to weak ferromagnetism. Symmetry suggests that the direct coupling between the ferromagnetism and ferroelectricity is mediated by DM interactions that exist only in the ferroelectric phase, controlling both the spiral rotation and the canting of the commensurate structure. The coupling between ferromagnetism and ferroelectricity permits to expect the existence of electricdipole active magnetic excitations in this system.

As for theoretical models, a detailed analysis of electromagnons in ferrimagnets was carried out only for the case of tetragonal $\mathrm{Mn}_{2} \mathrm{Sb}[92,93]$. Neutron diffraction experiments revealed that magnetic structure of $\mathrm{Mn}_{2} \mathrm{Sb}$ can be imagined as two ferromagnetically ordered (001) planes of MnI and MnII ions with different magnetic moments. The magnetic moments of $\mathrm{MnI}$ and $\mathrm{MnII}$ layers are antiparallel and are not coupled by inversion (two sublattices $a$ and $c$ subsystems). As it follows from the theoretical considerations [92,93], in this ferrimagnet the electric-dipole active excitations are vibrations of odd regarding to inversion antiferromagnetic vectors (as in the case of $\alpha-\mathrm{Fe}_{2} \mathrm{O}_{3}$ ). There are two proper frequencies of electromagnons corresponding two types of the $a$ and $c$ sublattices magnetization oscillation. As predicted, these elementary excitations could be detected as magnetic resonances in the dielectric susceptibility $\alpha_{i j}(\omega)$ of the system. Undoubtedly, the experimental testing is needed to verify the existence of the electric-dipole active magnons in $\mathrm{Mn}_{2} \mathrm{Sb}$. Unfortunately, the compound demonstrates a metallic-type conductivity, which will cause additional difficulties for detection of electromagnons.

\section{Conclusions}

In the above sections, only a little part of systems with electric-dipole active magnetic excitations has been reviewed. The author omitted the discussion of multiferroic properties and, accordingly, electromagnons modes detected in systems where the coupling mechanism between magnetic and electric properties is not uniquely identified and is under discussion. Concerning theoretical investigations, electromagnons in rare-earth orthoferrites $\mathrm{RFeO}_{3}$ and orthochromites $\mathrm{RCrO}_{3}$ crystallizing into crystallographic group $D_{2 h}^{16}=P b n m$ are also predicted. In these compounds, a primitive magnetic cell contains four sublattices of $d$-subsystem ( $\mathrm{Fe}$ or $\mathrm{Cr}$ ) and four rare-earth sublat- tices, and thus, any consistent description of these materials would be rather cumbersome. The interested reader is referred to Refs. 65,89 . The discussion of electric-dipole active nuclear magnetic excitations can be found in the original reports $[93,94]$.

In conclusion, recent rediscoveries of the so-called multiferroic materials, where the magnetic order and ferroelectric polarization coexist, have led to a surge of interest in this rather unusual class of materials. Intense research activities on these systems are partly motivated by the pure desire to understand the fundamental questions. At the same time, the immense potential for future applications, e.g., in spin wave electronics $[95,96]$, have made these multiferroic compounds one of the most sought-after recent topics in material science. The common studies of the magnon excitations are based on coupling between magnetic system and magnetic components of microwave field. However, this method is effective only for excitation of long-wave (acoustic) vibrations. Only recently the possibility to generate the exchange magnetic excitations was experimentally demonstrated for $\mathrm{Y}_{3} \mathrm{Fe}_{5} \mathrm{O}_{12}$ by method of parametric pumping [97]. In contrast, magnetic resonances driven by the electric component of field give a direct mode to effectively control of the magnetic sate by external electric field and to transform one type of excitation into another exactly in terahertz time-domain. Those are of crucial importance for the magnon-based spintronics devices as only short-wavelength exchange magnons allow signal processing at nanoscales.

1. G.W. Ludwig and H.H. Woodbury, Phys. Rev. Lett. 7, 240 (1961).

2. G.W. Ludwig and F.S. Ham, Phys. Rev. Lett. 8, 210 (1962).

3. A.B. Roitsin, Sov. Phys. Usp. 14, 766 (1972).

4. M.D. Glinchuk, V.G. Grachov, M.F. Deipen, A.B. Roitsin, and L.A. Syslin, Electric Effects in Radiospectroscopy, Nauka, Moskva (1981) (in Russian).

5. V.B. Mims, Electric-Field Effect in Paramagnetic Resonance, Naukova dumka, Kyiv (1982) (in Russian).

6. R. Ulbricht, E. Hendry, J. Shan, T.F. Heinz, and Bonn, Rev. Mod. Phys. 83, 543 (2011).

7. V.L. Bratman, A.G. Litvak, and E.V. Suvorov, Phys. Usp. 54, 837 (2011).

8. H. Schmid, Ferroelectrics 62, 317 (1994).

9. A. Pimenov, A.A. Mukhin, V.Yu. Ivanov, V.D. Travkin, A.M. Balbashov, and A. Loidl, Nature Phys. 2, 97 (2006).

10. A. Pimenov, T. Rudolf, F. Mayr, A. Loidl, A.A. Mukhin, and A.M. Balbashov, Phys. Rev. B 74, 100403(R) (2006).

11. V.G. Bar'yakhtar and I.E. Chupis. Sov. Phys. Solid State 10, 3547 (1968); ibid. 11, 2628 (1970).

12. V.G. Bar'yakhtar and I.E. Chupis. Int. J. Magn. 5, 337 (1974).

13. G.A. Smolenskii and I.E. Chupis, Sov. Phys. Usp. 25, 475 (1982). 
14. T. Kimura, T. Goto, H. Shintani, K. Ishizaka, T. Arima, and Y. Tokura, Nature (London) 426, 55 (2003).

15. A.A. Volkov, Yu.G. Goncharov, G.V. Kozlov, S.P. Lebedev, and A.M. Prochorov, Infrared Phys. 25, 369 (1985).

16. H. Katsura, N. Nagaosa, and A.V. Balatsky, Phys. Rev. Lett. 95, 057205 (2005).

17. M. Mostovoy, Phys. Rev. Lett. 96, 067601 (2006).

18. I.A. Sergienko and E. Dagotto, Phys. Rev. B 73, 094434 (2006).

19. S. Picozzi, K. Yamauchi, B. Sanyal, I.A. Sergienko, and E. Dagotto, Phys. Rev. Lett. 99, 227201 (2007).

20. V.Yu. Pomjakushin, M. Kenzelmann, A. Donni, A.B. Harris, T. Nakajima, S. Mitsuda, M. Tachibana, L. Keller, J. Mesot, H. Kitazawa, and E. Takayama-Muromachi, New J. Phys. 11, 043019 (2009).

21. S. Ishiwata, Y. Kaneko, Y. Tokunaga, Y. Taguchi, T. Arima, and Y. Tokura, Phys. Rev. B 81, 100411 (2010).

22. R.D. Johnson, L.C. Chapon, D.D. Khalyavin, P. Manue, P.G. Radaelli, and C. Martin, Phys. Rev. Lett. 108, 067201 (2012).

23. H. Katsura, A.V. Balatsky, and N. Nagaosa, Phys. Rev. Lett. 98, 027203 (2007).

24. D. Senff, P. Link, K. Hradil, A. Hiess, L.P. Regnault, Y. Sidis, N. Aliouane, D.N. Argyriou, and M. Braden, Phys. Rev. Lett. 98, 137206 (2007).

25. R. Valdès Auilar, A.B. Sushkov, C.L. Zhang, Y.J. Choi, S.W. Cheong, and H.D. Drew, Phys. Rev. B 76, 060404(R) (2007).

26. A. Pimenov, A. Loidl, A.A. Mukhin, V.D. Travkin, V.Yu. Ivanov, and A.M. Balbashov, Phys. Rev. B 77, 014438 (2008).

27. N. Kida, Y. Ikebe, Y. Takahashi, J.P. He, Y. Kaneko, Y. Yamasaki, R. Shimano, T. Arima, N. Nagaosa, and Y. Tokura, Phys. Rev. B 78, 104414 (2008).

28. D. Senff, P. Link, N. Aliouane, D.N. Argyriou, and M. Braden, Phys. Rev. B 77, 174419 (2008).

29. Y. Takahashi, N. Kida, Y. Yamasaki, J. Fujioka, T. Arima, R. Shimano, S. Miyahara, M. Mochizuki, N. Furukawa, and Y. Tokura, Phys. Rev. Lett. 101, 187201 (2008).

30. N. Kida, Y. Yamasaki, R. Shimano, T. Arima, and Y. Tokura, J. Phys. Soc. Jpn. 77, 123704 (2008).

31. A. Pimenov, A.M. Shuvaev, A.A. Mukhin, and A. Loidl, $J$. Phys.: Condens. Matter 20, 434209 (2008).

32. D. Senff, N. Aliouane, D.N. Argyriou, A. Hiess, L.P. Regnault, P. Link, K. Hradil, Y. Sidis, and M. Braden, J. Phys.: Condens. Matter 20, 434212 (2008).

33. N. Kida, Y. Yamasaki, J.P. He, Y. Kaneko, Y. Ikebe, Y. Takahashi, R. Shimano, T. Arima, N. Nagaosa, and Y. Tokura, J. Phys. Conf. Ser. 148, 012038 (2009).

34. R. Valdes Auilar, M. Mostovoy, A.B. Sushkov, C.L. Zhang, Y.J. Choi, S.-W. Cheong, and H.D. Drew, Phys. Rev. Lett. 102, 047203 (2009).

35. N. Kida, Y. Takahashi, J.S. Lee, R. Shimano, Y. Yamasaki, Y. Kaneko, S. Miyahara, N. Furukawa, T. Arima, and Y. Tokura. J. Opt. Soc. Am. B 26, A35 (2009).
36. A. Pimenov, A. Shuvaev, A. Loidl, F. Schrettle, A.A. Mukhin, V.D. Travkin, V.Yu. Ivanov, and A.M. Balbashov, Phys. Rev. Lett. 102, 107203 (2009).

37. J.S. Lee, N. Kida, S. Miyahara, Y. Yamasaki, Y. Takahashi, R. Shimano, N. Furukawa, and Y. Tokura, Phys. Rev. B 79, 180403(R) (2009).

38. S. Pailhès, X. Fabrèges, L.P. Règnault, L. Pinsard-Godart, I. Mirebeau, F. Moussa, M. Hennion, and S. Petit, Phys. Rev. $B$ 79, 134409 (2009).

39. Y. Takahashi, Y. Yamasaki, N. Kida, Y. Kaneko, T. Arima, R. Shimano, and Y. Tokura, Phys. Rev. B 79, 214431 (2009).

40. A.M. Shulaev, V.D. Travkin, V.Yu. Ivanov, A.A. Mukhin, and A. Pimenov, Phys. Rev. Lett. 104, 097202 (2010).

41. A.M. Shulaev, J. Hemberger, D. Niermann, F.F. Schrettle, A. Loidl, V.Yu. Ivanov, V.D. Travkin, A.A. Mukhin, and A. Pimenov, Phys. Rev. B 82, 174417 (2010).

42. M.P.V. Stenberg and R. de Sousa, Phys. Rev. B 80, 094419 (2009).

43. M. Mochizuki, N. Furukawa, and N. Nagaosa, Phys. Rev. Lett. 104, 177206 (2010).

44. Y. Takahashi, S. Ishiwata, S. Miyahara, Y. Kaneko, N. Furukawa, Y. Taguchi, R. Shimano, and Y. Tokura, Phys. Rev. B 81, 100413(R) (2010).

45. M. Cazayous, Y. Gallais, A. Sacuto, R. de Sousa, D. Lebeugle, and D. Colson, Phys. Rev. Lett. 101, 037601 (2008).

46. M.K. Singh, R.S. Katiyar, and J.F. Scott, J. Phys.: Condens. Matter 20, 252203 (2008).

47. E. Golovenchits and V. Sanina, J. Phys.: Condens. Matter 16, 4325 (2004).

48. A.B. Sushkov, R.V. Aguilar, S. Park, S.-W. Cheong, and H.D. Drew, Phys. Rev. Lett. 98, 027202 (2007).

49. C. Fang and J. Hu, Euro. Phys. Lett. 82, 57005 (2008).

50. S. Seki, N. Kida, S. Kumakura, R. Shimano, and Y. Tokura. arXiv:1008.4709v1 (2010).

51. S. Seki, N. Kida, S. Kumakura, R. Shimano, and Y. Tokura, Phys. Rev. Lett. 105, 097207 (2010).

52. T. Nakajima, A. Suno, S. Mitsuda, N. Terada, S. Kimura, K. Kaneko, and Hiroki Yamauchi, Phys. Rev. B 84, 184401 (2011).

53. I. Kèzsmárki, N. Kida, H. Murakawa, S. Bordács, Y. Onose, and Y. Tokura, Phys. Rev. Lett. 106, 057403 (2011).

54. V.G. Bar'yakhtar, V.A. L'vov, and D.A. Yablonskii, JETP Lett. 37, 565 (1983)

55. F. Kagawa, M. Mochizuki, Y. Onose, H. Murakawa, Y. Kaneko, N. Furukawa, and Y. Tokura, Phys. Rev. Lett. 102, 057604 (2009).

56. F. Kubel and H. Schmid, Acta Crystallogr. B 46, 698 (1990).

57. D. Lebeugle, D. Colson, A. Forget, and M. Viret, Appl. Phys. Lett. 91, 22907 (2007).

58. C. Jia, S. Onoda, N. Nagaosa, and J.H. Han, Phys. Rev. B 76, 144424 (2007).

59. T. Moria, J. Appl. Phys. 39, 1042 (1968).

60. S. Miyahara and N. Furukawa, arXiv:0811.4082 (2008). 
61. V.G. Bar'yakhtar, V.N. Krivoruchko, and D.A. Yablonskii, Green's Functions in the Theory of Magnetism, Naukova dumka, Kyiv (1984) (in Russian).

62. M. Mochizuki, N. Furukawa, and N. Nagaosa, Phys. Rev. Lett. 105, 037205 (2010).

63. D.A. Yablonskii and V.N. Krivoruchko, Fiz. Nizk. Temp. 14, 656 (1988) [Sov. J. Low Temp. Phys. 14, 363 (1988)].

64. V.N. Krivoruchko and D.A. Yablonskii, Sov. Phys. JETP 67, 1886 (1988); arXiv: 1203.2731 (2012).

65. D.A. Yablonskii and V.N. Krivoruchko, Fiz. Tverd. Tela 30, 3069 (1988).

66. V.V. Eremenko, V.N. Krivoruchko, N.M. Lavrinenko, and D.A. Yablonskii, Fiz. Tverd. Tela 30, 3605 (1988).

67. V.N. Krivoruchko, N.M. Lavrinenko, and D.A. Yablonskii, Fiz. Tverd. Tela 31, 69 (1989).

68. V.N. Krivoruchko, Fiz. Tverd. Tela 32, 338 (1990).

69. V.N. Krivoruchko, T.E. Solovyeva, and D.A. Yablonskii, Optics and Spectroscopy 68, 550 (1990).

70. V.N. Krivoruchko and T.E Primak, Fiz. Tverd. Tela, 33, 3205 (1991).

71. V.G. Bar'yakhtar, I.M. Vitebskii, Yu.G. Pashkevich, V.L. Sobolev, and V.V. Tarasenko, Sov. Phys. JETP 60, 587 (1984).

72. I.M. Vitebskii and N.M. Lavrinenko, Fiz. Nizk. Temp. 12, 1193 (1986) [Sov. J. Low Temp. Phys. 12, 672 (1986)].

73. V.V. Druzhinin, Sov. Phys. Solid State 7, 764 (1965).

74. T. Moriya, J. Phys. Soc. Jpn. 23, 490 (1967).

75. V.V. Eremenko and A.I. Belyaeva, Sov. Phys. Usp. 12, 320 (1969).

76. V.V. Eremenko, Introduction to Optical Spectroscopy of Magnets, Naukova dumka, Kyiv (1975) (in Russian).

77. I.E. Dzyaloshinskii, Sov. Phys. JETP 5, 1259 (1957).

78. R. Hornreich and S. Shtrikman, Phys. Rev. 161, 506 (1967).

79. I.E. Dzyaloshinskii, Sov. Phys. JETP 10, 628 (1960).

80. D.N. Astrov, Sov. Phys. JETP 13, 729 (1961).

81. L.D. Landau and I.M. Lifshitz, Electrodynamics of Continuous Media, Pergamon (1984).

82. G.T. Rado, Int. J. Magn. 6, 121 (1974).
83. N. Kida, D. Okuyama, S. Ishiwata, Y. Taguchi, R. Shimano, K. Iwasa, T. Arima, and Y. Tokura, Phys. Rev. B 80, 220406(R) (2009).

84. N. Kida, S. Kumakura, S. Ishiwata, Y. Taguchi, and Y. Tokura, Phys. Rev. B 83, 064422 (2011).

85. N. Momozawa, Y. Yamaguchi, and M. Mita, J. Phys. Soc. Jpn. 55, 1350 (1986).

86. P.D. Roders, Y.J. Choi, E.C. Standard, T.D. Kang, K.H. Ahn, A. Dubroka, P. Marsik, Ch. Wang, C. Bernhard, S. Park, S.-W. Cheong, M. Kotelyanskii, and A.A. Sirenko, Phys. Rev. B 83, 174407 (2011).

87. G.A. Babushkin, A.K. Zvezdin, R.Z. Levitin, V.N. Orlov, and A.I. Popov, Sov. Phys. JETP 58, 792 (1983).

88. J.B. Gruber, D.K. Sardar, R.M. Yow, U.V. Valiev, A.K. Mukhammadiev, V.Yu. Sokolov, I. Amin, K. Lengyel, I.S. Kachur, V.G. Piryatinskaya, and B. Zandi, J. Appl. Phys. 101, 023108 (2007).

89. A.K. Zvezdin and A.A. Mukhin, JETP Lett. 88, 581 (2008). (in Russian).

90. K. Kamazawa, D. Louca, R. Morinaga, T.J. Sato, Q. Huang, J.R.D. Copley, and Y. Qiu, Phys. Rev. B 78, 064412 (2008).

91. J.S. White, T. Honda, K. Kimura, T. Kimura, Ch. Niedermayer, O. Zaharko, A. Poole, B. Roessli, and M. Kenzelmann, Phys. Rev. Lett. 108, 077204 (2012).

92. I.F. Mirsaev and E.A. Turov, JETP 121, 419 (2002) (in Russian).

93. E.A. Turov and V.V. Nikolaev, Physics Uspekhi 48, 431 (2005).

94. M.I. Kurkin, I.F. Mirsaev, and E.A. Turov, JETP 125, 1144 (2004) (in Russian).

95. K. Uchida, J. Xiao, H. Adachi, J. Ohe, S. Takahashi, J. Ieda, T. Ota, Y. Kajiwara, H. Umezawa, H. Kawai, G.E.W. Bauer, S. Maekawa, and E. Saitoh, Nature Materials 9, 894 (2010).

96. Y. Kajiwara, K. Harii, S. Takahashi, J. Ohe, K. Uchida, M. Mizuguchi, H. Umezawa, H. Kawai, K. Ando, K. Takanashi, S. Maekawa, and E. Saitoh, Nature (London) 464, 262 (2010).

97. C.W. Sandweg, Y. Kajiwara, A.V. Chumak, A.A. Serga, V.I. Vasyuchka, M.B. Jungfleisch, E. Saitoh, and B. Hillebrands, Phys. Rev. Lett. 106, 216601 (2011). 